\title{
Ferroptosis, radiotherapy, and combination therapeutic strategies
}

\author{
Guang Lei ${ }^{1,2}$, Chao Mao², Yuelong Yan², Li Zhuang ${ }^{2}$, Boyi Gan ${ }^{2,3 \bowtie}$ \\ ${ }^{1}$ Department of Radiation Oncology, Hunan Cancer Hospital and The Affiliated Cancer Hospital of Xiangya School of \\ Medicine, Central South University, Changsha 410013, China \\ 2 Department of Experimental Radiation Oncology, The University of Texas MD Anderson Cancer Center, Houston, TX 77030, \\ USA \\ ${ }^{3}$ The University of Texas MD Anderson UTHealth Graduate School of Biomedical Sciences, Houston, TX, USA \\ $\triangle$ Correspondence: bgan@mdanderson.org (B. Gan)
}

Received February 18, 2021 Accepted March 29, 2021

\begin{abstract}
Ferroptosis, an iron-dependent form of regulated cell death driven by peroxidative damages of polyunsaturated-fatty-acid-containing phospholipids in cellular membranes, has recently been revealed to play an important role in radiotherapy-induced cell death and tumor suppression, and to mediate the synergy between radiotherapy and immunotherapy. In this review, we summarize known as well as putative mechanisms underlying the crosstalk between radiotherapy and ferroptosis, discuss the interactions between ferroptosis and other forms of regulated cell death induced by radiotherapy, and explore combination therapeutic strategies targeting ferroptosis in radiotherapy and immunotherapy. This review will provide important frameworks for future investigations of ferroptosis in cancer therapy.
\end{abstract}

KEYWORDS ferroptosis, lipid peroxidation, GPX4, SLC7A11, radiotherapy, immunotherapy, radiosensitization, combination therapy

\section{INTRODUCTION}

Regulated cell death (RCD), such as apoptosis, is a recognized hindrance to tumorigenesis. Consequently, cancer cells gradually evolve resistance to RCDs during tumor progression (Hanahan and Weinberg, 2011; Galluzzi et al., 2018; Green, 2019). Ferroptosis is a recently identified form of RCD driven by iron-dependent lipid peroxidation, which is distinct from other RCDs, such as apoptosis, autophagy and necroptosis, in morphology and mechanisms (Dixon et al., 2012; Stockwell et al., 2017). Inhibitors for these other RCDs generally are ineffective in blocking ferroptosis (Dixon et al., 2012) (although in some contexts ferroptosis is also considered a form of autophagy-dependent cell death (Gao et al., 2016; Hou et al., 2016)). Morphologically, ferroptosis is neither characterized by typical apoptotic features, such as chromatin condensation and apoptotic body formation, nor by the formation of autophagosomes, a key feature of autophagy; instead, ferroptotic cells generally exhibit shrunken mitochondria with increased mitochondrial membrane density and diminished mitochondrial cristae (Dixon et al., 2012; Stockwell et al., 2017). Mechanistically, polyunsaturated-fatty-acid-containing phospholipids (PUFAPLs) in cellular membranes are susceptible for peroxidation under iron- and reactive oxygen species (ROS)-rich conditions. A toxic buildup of such lipid peroxides in cellular membranes eventually damages membrane integrity, leading to ferroptotic cell death (Stockwell et al., 2017).

Cells have evolved diverse array of ferroptosis defense systems, including glutathione peroxidase 4 (GPX4)-dependent and -independent systems, to detoxify lipid peroxides, thereby preventing their accumulation to lethal levels and maintaining cell survival (Stockwell et al., 2017; Zheng and Conrad, 2020). Accordingly, inactivation of such defense systems by genetic or pharmacological approaches provokes ferroptosis (Liang et al., 2019). Importantly, ferroptosis is not only associated with multiple pathologic conditions and diseases, but has also been identified as a natural barrier to cancer development. Inactivation of some tumor suppressors, such as tumor protein p53 (p53) and BRCA1 associated protein-1 (BAP1), promotes tumor development at least partly via suppressing tumor ferroptosis (Jiang et al., 2015; Zhang et al., 2018; Zhang et al., 2019b; Stockwell et al., 2020). Likewise, ferroptosis was recently shown to play an 
important role in some cancer therapies, and inducing tumor ferroptosis has emerged as a promising strategy for cancer treatment (Hassannia et al., 2019).

Radiotherapy (RT), a common cancer treatment modality, uses targeted delivery of ionizing radiation (IR) to eradicate cancer cells (Delaney et al., 2005; Jaffray, 2012). IR penetrates the tumor field and induces both direct and indirect cellular effects. It directly induces various types of DNA damage, such as base damage, single strand breaks (SSBs), and double strand breaks (DSBs) (Baidoo et al., 2013). In addition, IR elicits radiolysis of cellular water and stimulates oxidative enzymes to generate highly reactive $\mathrm{OH}$ - radicals as well as other ROS, including $\mathrm{O}_{2}^{\circ-}$ and $\mathrm{H}_{2} \mathrm{O}_{2}$, which can subsequently attack nucleic acids, lipids, and proteins in a dose-dependent manner (Azzam et al., 2012; Reisz et al., 2014). These direct and indirect effects together trigger adverse cellular events in cancer cells, including cell cycle arrest, cellular senescence, and RCDs such as apoptosis; however, the potential role and mechanisms of other forms of RCD in RT remain to be further studied (Adjemian et al., 2020).

Recently studies revealed that IR induces potent ferroptosis and that ferroptosis represents an important part of RTmediated anticancer effects (Lang et al., 2019; Lei et al., 2020; Ye et al., 2020). In clinic, RT generally needs to be combined with chemotherapy, targeted therapies, or immunotherapy to eliminate cancer cells. Notably, ferroptosis has also been linked to the efficacy of some of abovementioned cancer therapies (Ma et al., 2016; Sun et al., 2016; Guo et al., 2018; Wang et al., 2019b). In the following sections, we first briefly review our current understanding of IR-induced signaling and cellular effects, as well as ferroptosis pathways and its inducers. We then discuss various aspects of IR-induced ferroptosis, including its known and other potential mechanisms, the role of ferroptosis modulators in radiosensitivity and RT-activated immune responses, potential interactions of ferroptosis with other IR-induced RCDs. Finally, we explore therapeutic implications of targeting ferroptosis in overcoming tumor radioresistance, the possibility of using ferroptosis regulators as potential predictive markers for RT efficacy, and the relevance of ferroptosis to RT combined with immunotherapy.

\section{IR-INDUCED SIGNALING AND CELLULAR EFFECTS}

Once IR induces DNA damage, ataxia telangiectasia mutated (ATM) and ataxia telangiectasia and Rad3 related (ATR) serine/threonine kinases rapidly detect these damages and induce complex signaling cascades known as DNA damage response (DDR) that activate the downstream checkpoint kinases $1 / 2$ (CHEK1/2), which then phosphorylate p53, among others, to arrest the cell cycle so that the damages in DNA can be corrected by DNA repair machineries (Huang and Zhou, 2020). The ultimate fate of these cells is at least partly determined by the severity of IR-induced DNA damage: if the damage can be fully repaired, cells survive and reenter into cell cycle; in contrast, irreparable or improperly repaired DNAs in the genome will trigger senescence (a permanent state of cell cycle arrest), apoptosis, or other forms of RCD, the exact outcome of which is often related to the radiation dose, linear energy transfer (LET), cell types, and the status of key cellular factors, including p53 (Maier et al., 2016).

Regarding p53's function in RT, p53 is stabilized and activated by $\mathrm{RT}$, and then operates as a transcription factor to govern the transcription of diverse genes such as cyclin dependent kinase inhibitor 1A (CDKN1A/p21), plasminogen activator inhibitor-1 (PAI-1), and promyelocytic leukemia protein (PML), which function to permanently arrest the cell cycle, thereby contributing to senescence (Bieging et al., 2014). Senescence is the terminus of most irradiated normal cells and a barrier for cancer development (Braig et al., 2005; Maier et al., 2016). Since $p 53$ is frequently mutated in cancer cells, other senescence checkpoints, such as the p16-retinoblastoma (RB) pathway, are also responsible for eliminating cancer cells upon IR (Sabin and Anderson, 2011). Notably, some of the senescent cells may also eventually undergo apoptosis. It has been indicated that the more potent and prolonged the activation of p53 by IR is, the more likely cells will undergo apoptosis rather than senescence (Vousden, 2000; Mijit et al., 2020). To induce apoptosis, p53 activation upregulates the expression of genes such as p53 upregulated modulator of apoptosis (PUMA), BCL2-Associated $X(B A X)$, and phorbol-12-myristate-13acetate-induced protein 1 (NOXA), leading to irreversible mitochondrial outer membrane permeability (MOMP), which releases cytochrome $C$ and activates the caspase-9/3/7 pathway, thereby inducing intrinsic apoptosis (Aubrey et al., 2018); alternatively, p53 induces the death receptors FAS (CD95), death receptor 5 (DR5) and FAS ligands, ultimately activating caspase- 8 and its downstream effectors to trigger extrinsic apoptosis (Sheikh and Fornace, 2000).

RT can also induce other apoptosis-independent RCDs. Specifically, IR has been shown to induce autophagy or necroptosis in certain contexts. There exists a complex interaction between IR and autophagy (a cellular process wherein intracellular cargos are degraded in autophagosomes and recycled into the cytosol) (Hu et al., 2016). Multiple factors, such as ATM, AMP-activated protein kinase (AMPK), Sirtuin 1 (SIRT-1), and mitochondrial ROS, contribute to the induction of autophagy by IR, and autophagy can exert a pro-survival or pro-cell death function in IR-mediated cellular effects, depending on the context (Bristol et al., 2012; Hu et al., 2016). Therefore, the exact role of autophagy in radiosensitization remains somewhat controversial. Necropotosis is a caspase-independent RCD triggered by the phosphorylation-dependent activation of mixed lineage kinase domain like pseudokinase (MLKL) mediated by the receptor-interacting serine/threonine protein kinases 1/3 (RIPK1/3) complex. Recent studies suggest that IR can induce necropotosis in certain cancer cells, although 
necroptosis appears not to be the predominant RCD in response to IR (Nehs et al., 2011; Adjemian et al., 2020).

In addition, although mitotic catastrophe, a mechanism of abnormal mitosis-induced cell death, is a common cellular effect of RT, it is not strictly considered as an RCD (Galluzzi et al., 2018). Cells in mitotic catastrophe are almost unable to replicate, and the vast majority of cells eventually die, with only a small fraction resuming proliferation (Vakifahmetoglu et al., 2008). Finally, necrosis, as a non-RCD triggered by IR, is more commonly associated with the side effects of RT, such as cerebral or pulmonary radiation necrosis (Song and Colaco, 2018; Benveniste et al., 2019). In brief summary, IR can induce complex downstream signaling networks and trigger a diverse array of adverse cellular effects.

\section{FERROPTOSIS PATHWAYS AND INDUCERS}

The accumulation of iron-dependent lipid peroxides is the cornerstone of ferroptosis (Dixon et al., 2012; Stockwell et al., 2017; Zheng and Conrad, 2020). Under normal conditions, ferroptosis defense systems can detoxify lipid peroxides and maintain them at non-toxic levels. When ferroptosis-executing systems override ferroptosis defense systems (such as when ferroptosis defense systems become largely defective), lipid peroxides quickly accumulate to toxic levels in cellular membranes, triggering ferroptosis (Stockwell et al., 2020; Zheng and Conrad, 2020) (Fig. 1). In this section, we discuss ferroptosis defense systems (including both GPX4-dependent and -independent systems) and ferroptosis-executing systems (including PUFA-PL metabolism and peroxidation, and iron metabolism). To facilitate our later discussion on targeting ferroptosis in overcoming radioresistance, we will also introduce ferroptosis inducers (FINs, the compounds capable of inducing ferroptosis in cancer cells) in this section. We refer readers to other excellent reviews for more extensive introduction of ferroptosis mechanisms (Stockwell et al., 2017; Stockwell et al., 2020; Zheng and Conrad, 2020).

\section{GPX4-dependent system}

The solute carrier family 7 member 11-glutathione-GPX4 (SLC7A11-GSH-GPX4) signaling axis is believed to constitute the predominant ferroptosis defense system; indeed, ferroptosis was originally uncovered based on studies on this signaling axis (Dixon et al., 2012; Angeli et al., 2014; Dixon et al., 2014; Yang et al., 2014) (Fig. 1). SLC7A11 (also known as $\mathrm{xCT}$ ) is a core component of the cystine/glutamate antiporter system $\mathrm{x}_{\mathrm{c}}^{-}$, and mediates the antiporter activity of system $x_{c}^{-}$by importing extracellular cystine and exporting intracellular glutamate (Sato et al., 1999; Koppula et al., 2018). SLC7A11 takes up extracellular cystine and subsequently cystine is rapidly reduced to cysteine in cytosol through a nicotinamide adenine dinucleotide phosphate (NADPH)-consuming reduction reaction (Conrad and Sato, 2012; Koppula et al., 2018; Liu et al., 2020c; Liu et al., 2020d). Cysteine then serves as the rate-limiting precursor for the biosynthesis of GSH, a principle cofactor for GPX4 to detoxify lipid peroxides (Koppula et al., 2020). Blocking SLC7A11 transporter activity or depriving cystine in culture media induces potent ferroptosis in many cancer cells (Koppula et al., 2020). Notably, several tumor suppressors, including p53, BAP1, ADP-ribosylation factor (ARF), and Kelch-like ECH-associated protein 1 (KEAP1), promote ferroptosis by suppressing the expression or activity of SLC7A11 as part of their tumor suppressive activities (Jiang et al., 2015; Chen et al., 2017b; Fan et al., 2017; Zhang et al., 2018). Likewise, activating transcription factor 3 (ATF3) represses SLC7A11 expression by binding to the SLC7A11 promoter, boosting the sensitivity of cancer cells to ferroptosis (Wang et al., 2020). SLC7A11 can be induced under various stress conditions, such as oxidative stress and amino acid starvation, by stress-responsive transcription factors such as nuclear factor erythroid 2-related factor 2 (NRF2) and activating transcription factor 4 (ATF4), thereby protecting cells from ferroptosis under stress conditions (Habib et al., 2015; Chen et al., 2017a; Fan et al., 2017). SLC7A11 can also be regulated at posttranscriptional levels. For example, the cancer stem cell marker CD44 and the deubiquitinating enzyme OTU domain-containing ubiquitin aldehyde binding protein 1 (OTUB1) promote ferroptosis resistance through stabilizing SLC7A11 (Ishimoto et al., 2011; Chew et al., 2017; Liu et al., 2019b).

It should be noted that in some cancer cells the transsulfuration pathway can supply a portion of intracellular cysteine for GSH synthesis through de novo synthesis of cysteine (Zhu et al., 2019), and enzymes that are involved in or regulated by the transsulfuration pathway (Fig. 1), such as cystathionine $\beta$-synthase (CBS) and cysteinyl-tRNA synthetase (CARS), can modulate the susceptibility of cancer cells to ferroptosis (Hayano et al., 2016; Wang et al., 2018). However, it is believed that intracellular cysteine derived from the transsulfuration pathway generally is not sufficient to cope with the high levels of oxidative stress to which cancer cells are exposed, and therefore most cancer cells still rely primarily on the acquisition of cysteine from the extracellular milieu via SLC7A11 (Chio and Tuveson, 2017; Koppula et al., 2020).

GPX4 utilizes $\mathrm{GSH}$ as its cofactor to reduce $\mathrm{PL}$ hydroperoxides to non-toxic PL alcohols, thereby maintaining the integrity of PL bilayers and preventing ferroptosis (Stockwell et al., 2017; Stockwell et al., 2020) (Fig. 1). GPX4 inactivation, pharmacologically or genetically, leads to drastic accumulation of toxic lipid peroxides and triggers ferroptosis (Angeli et al., 2014; Yang et al., 2014). Regarding GPX4's role in cancer, GPX4 is overexpressed in a variety of cancers, and Gpx4+/- mice exhibit delayed lymphomagenesis compared with their wild-type counterparts (Ran et al., 2007; Zhang et al., 2020). Certain cancer cells, such as drug-tolerant persister cancer cells or therapy-resistant highmesenchymal ones, are highly dependent on GPX4 activity, thereby exposing potential vulnerabilities for therapeutic 


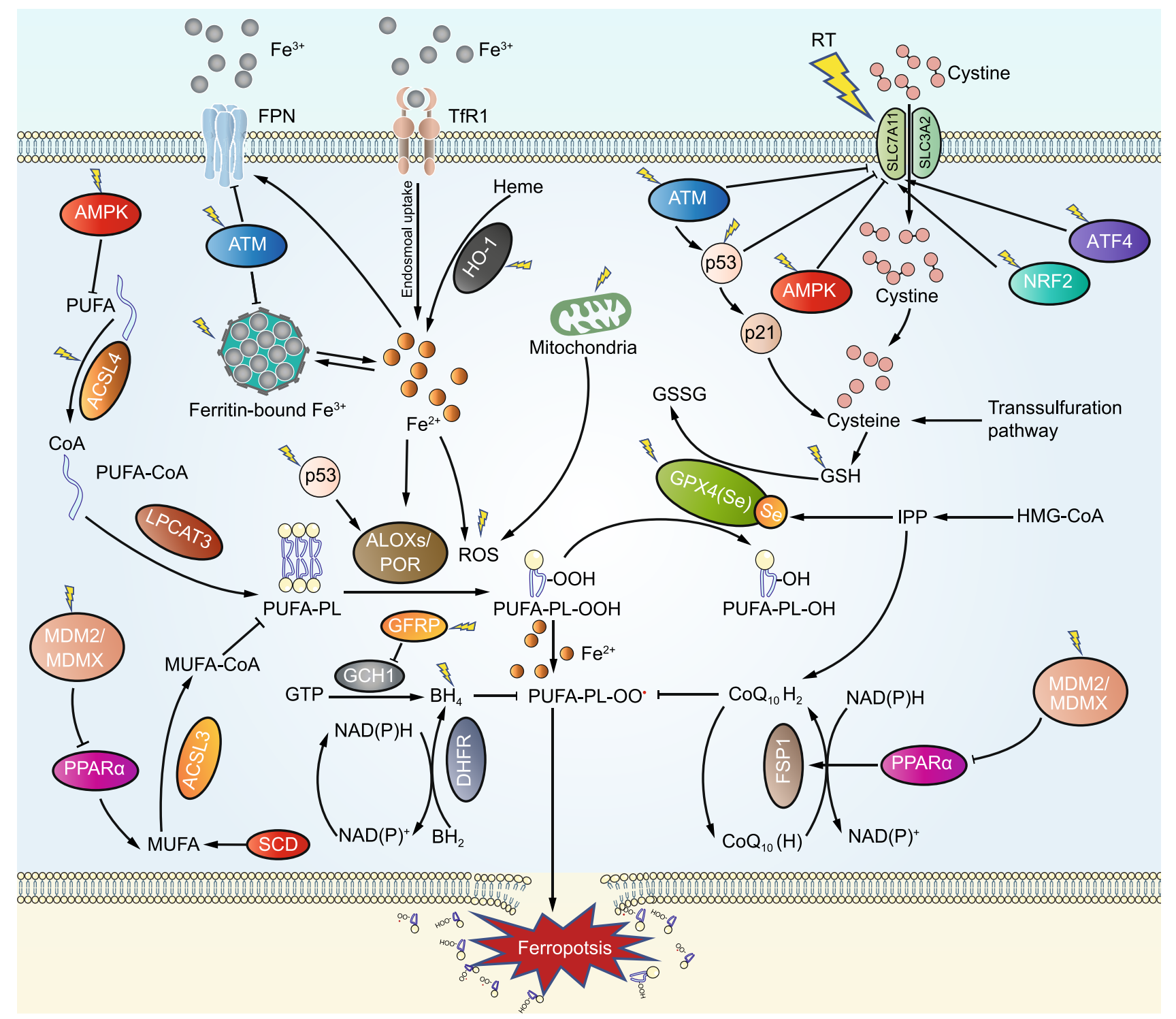

Figure 1. The ferroptosis signaling pathway and ferroptosis regulators with known and potential relevance to radiotherapy. Ferroptosis is driven by the accumulation of PUFA-PL peroxides, whose generation is facilitated by iron metabolism, PUFA-PL synthesis and peroxidation. Ferroptosis is counteracted by ferroptosis defense systems including the SLC7A11-GSH-GPX4, NAD(P) $\mathrm{H}-\mathrm{FSP} 1-\mathrm{CoQ}$, and $\mathrm{GCH} 1-\mathrm{BH} 4$ axes. Several regulators in the ferroptosis pathway that are modulated by radiotherapy are also highlighted. These regulators either have been confirmed to play roles or potentially might have roles in radiotherapy-induced ferroptosis.

targeting (Ran et al., 2007; Hangauer et al., 2017; Viswanathan et al., 2017). GPX4 is a selenoprotein; the selenocysteine $(\mathrm{Sec})$ residue in GPX4 is required for its antiferroptosis activity (Angeli and Conrad, 2018; Ingold et al., 2018). Selenium supplementation not only promotes GPX4 protein synthesis, but also drives its transcription, while perturbation of the mevalonate pathway impairs translation of selenoproteins (including GPX4), thereby sensitizing cells to ferroptosis (Ingold et al., 2018; Alim et al., 2019; Conrad and Proneth, 2020). Together, SLC7A11-mediated cystine uptake, GSH biosynthesis, and GPX4 activity constitute a robust ferroptosis defense system that keeps lipid hydroperoxides at levels below the toxic threshold to maintain cell survival. 


\section{GPX4-independent defense systems}

The $\mathrm{NAD}(\mathrm{P}) \mathrm{H}$-ferroptosis suppressor protein 1-ubiquinone [NAD $(\mathrm{P}) \mathrm{H}-\mathrm{FSP} 1-\mathrm{CoQ}$ ] signaling axis is a recently established ferroptosis defense system that operates in parallel to the SLC7A11-GSH-GPX4 axis (Bersuker et al., 2019; Doll et al., 2019) (Fig. 1). Derived from the mevalonate pathway and mainly synthesized in mitochondria, CoQ is not only an important element of the mitochondrial electron transport chain (ETC), but its reduced form, ubiquinol $\left(\mathrm{CoQH}_{2}\right)$, also acts as a potent lipophilic antioxidant (Frei et al., 1990; Duberley et al., 2014; Shimada et al., 2016). FSP1, also known as apoptosisinducing factor-associated mitochondrial-associated protein 2 (AIFM2), was previously suggested to participate in inducing apoptosis (Wu et al., 2002), but its role in apoptosis is complicated and somewhat controversial (Vařecha et al., 2007; Yang et al., 2011; Kaku et al., 2015; Nguyen et al., 2020). FSP1 functions as an oxidoreductase of CoQ (Marshall et al., 2005; Elguindy and Nakamaru-Ogiso, 2015). Recent studies revealed that FSP1 localizes on the plasma membrane and reduces $\mathrm{CoQ}$ to $\mathrm{CoQH}_{2}$ by consuming $\mathrm{NAD}(\mathrm{P}) \mathrm{H}$, and $\mathrm{CoQH}_{2}$ subsequently inhibits ferroptosis by trapping lipophilic free radicals; consequently, the blockade of $\mathrm{CoQ}$ biosynthesis pathway abolishes FSP1's ability to suppress ferroptosis (Bersuker et al., 2019; Doll etal., 2019). Importantly, the NAD(P) $\mathrm{H}-\mathrm{FSP} 1-\mathrm{CoQ}$ axis acts as an independent system in concert with the SLC7A11-GSH-GPX4 system to protect cells from ferroptosis. FSP1 has been considered as a p53-responsive gene (Horikoshi et al., 1999; Ohiro et al., 2002; Wu et al., 2004); however, recent studies demonstrated that FSP1 expression is not affected by p53 activator nutlin-3 or doxorubicin (Bersuker et al., 2019; Doll et al., 2019). Furthermore, a few studies have linked the regulation of FSP1 to cAMP-response-elementbinding protein (CREB) (Nguyen et al., 2020) or mouse double minute 2 homolog/murine double minute $X$ (MDM2/MDMX) complex (Venkatesh et al., 2020), although the exact relevance and the biological contexts of these regulations to ferroptosis remain to be further investigated.

Finally, tetrahydrobiopterin $\left(\mathrm{BH}_{4}\right)$ and its rate-limiting enzyme guanosine triphosphate cyclohydrolase 1 (GCH1) were recently identified as an alternative ferroptosis defense system independent of GPX4 (Kraft et al., 2019; Soula et al., 2020) (Fig. 1). $\mathrm{BH}_{4}$ is a robust radical-trapping antioxidant in cellular membranes and is capable of promoting the regeneration of $\mathrm{CoQH}_{2}$ and $\alpha$-tocopherol to counteract lipid peroxidation and ferroptosis (Crabtree et al., 2009; Kraft et al., 2019; Soula et al., 2020). $\mathrm{BH}_{4}$ is regenerated from its oxidized form boron dihydride $\left(\mathrm{BH}_{2}\right)$ via dihydrofolate reductase (DHFR); consequently, inactivation of DHFR significantly increases cellular vulnerability to ferroptosis (Soula et al., 2020).

\section{PUFA-PL synthesis and peroxidation}

Free PUFAs, such as arachidonic acids (AAs) and adrenic acids (AdAs), are catalyzed mainly by acyl coenzyme $A$ synthetase long chain family member 4 (ACSL4) to produce their acyl coenzyme $A$ (CoA) derivatives (such as AA/AdACoA). Subsequently, these PUFA-CoAs are processed to form lysophospholipids (LysoPLs) and further incorporated into PLs (such as AA-PE and AdA-PE) by lysophosphatidylcholine acyltransferase 3 (LPCAT3) and other enzymes (Fig. 1). Correspondingly, ablation of ACSL4 or LPCAT3 suppresses PUFA-PL synthesis and dramatically promotes ferroptosis resistance (Dixon et al., 2015; Doll et al., 2017; Kagan et al., 2017). In addition, energy stress (a metabolic stress condition with ATP depletion) activates AMPK, which suppresses acetyl-CoA carboxylase (ACC, which converts acetyl-CoA to malonyl-CoA) and reduces PUFA-PL levels (likely because malonyl-CoA is required for AA or AdA synthesis), resulting in ferroptosis blockade (Lee et al., 2020; Li et al., 2020) (Fig. 1).

Due to the presence of bis-allylic moieties in PUFAs, PUFA-PLs are particularly vulnerable to peroxidation (Conrad and Pratt, 2019). Lipid peroxidation is believed to occur through both enzyme-mediated reactions and enzymatic independent reactions known as autoxidation, wherein lipid peroxides can be generated through free radical chain reactions which require iron and oxygen (Conrad and Pratt, 2019). Regarding the enzymes that drive lipid peroxidation, while lipid peroxidation was initially proposed to be mediated by lipoxygenases (ALOXs) (Yang et al., 2016), the role of ALOXs in lipid peroxidation was subsequently challenged (Shah et al., 2018), and more recent studies revealed that, at least in most cancer cell lines, cytochrome P450 oxidoreductase (POR) appears to play a more dominant role in mediating lipid peroxidation (Yan et al., 2020; Zou et al., 2020b) (Fig. 1).

Other types of PLs are also involved in ferroptosis regulation. Recently PUFA-containing ether PLs (PUFA-ePLs) were found to act as an alternative substrate for lipid peroxidation (Zou et al., 2020a). In addition, supplementation of certain exogenous monounsaturated fatty acids (MUFAs) can displace PUFAs from PLs located in cellular membranes and render cells less susceptible to peroxidation, thereby attenuating ferroptosis (Magtanong et al., 2019). MUFA biosynthesis is mediated by stearoyl coenzyme A desaturase (SCD), and its incorporation into PLs requires acyl coenzyme A synthetase long chain family member 3 (ACSL3); correspondingly, SCD and ACSL3 have been shown to protect cells against ferroptosis (Paton and Ntambi, 2009; Magtanong et al., 2019; Tesfay et al., 2019) (Fig. 1).

\section{Iron metabolism}

The labile iron generates free radicals and mediates lipid peroxidation through Fenton reaction (Ayala et al., 2014). Iron chelation by desferoxamine (DFO) blocks ferroptosis (therefore its name "ferroptosis"), whereas increases in labile iron levels sensitizes cells to ferroptosis, establishing that iron is fundamental to ferroptosis (Dixon et al., 2012; Kim et al., 2016). Labile iron pool is primarily maintained by 
proteins responsible for its uptake, storage, and export (Fig. 1). Iron uptake relies primarily on transferrin receptor 1 (TFR1), which transports ferritin-bound iron into cells via receptor-mediated endocytosis; notably, TFR1 was also recently identified as a biomarker for ferroptosis (Anderson and Vulpe, 2009; Gao et al., 2015; Feng et al., 2020). Iron is principally stored in ferritin in the form of Fe (III) (inert iron), which is not involved in lipid peroxidation; therefore, the abundance of ferritin, especially ferritin heavy chain (FTH1), is critical for ferroptosis suppression (Mumbauer et al., 2019). Ferritinophagy, the autophagic degradation of ferritin, promotes the release of iron stored in ferritin into the labile iron pool, thereby sensitizing cells to ferroptosis (Gao et al., 2016). Iron is mainly exported by ferroportin 1 (FPN1), and iron export is further facilitated by prominin2, which regulates the formation of ferritin-containing multivesicular bodies and exosomes; correspondingly, inhibition of these proteins drives ferroptosis (Geng et al., 2018; Brown et al., 2019). Moreover, several enzymes essential for lipid peroxidation, such as ALOXs and POR, are iron-dependent, and Fe (II) that is not bound to these enzymes further accelerates the propagation of peroxides during lipid peroxidation, leading to extensive ferroptosis (Wenzel et al., 2017; Shah et al., 2018; Zou et al., 2020b) (Fig. 1).

\section{Ferroptosis inducers}

Several classes of FINs have been identified and developed, including class I FINs that inhibit SLC7A11 activity and deplete GSH, class II FINs that directly inhibit GPX4 activity by covalently binding to selenocysteine at the active site of GPX4, class III FINs that activate squalene synthase (SQS), thereby indirectly depleting both $\mathrm{CoQ}$ and GPX4, as well as other types of FINs (Hassannia et al., 2019). Besides, various nanomaterials have been exploited to induce ferroptosis locally (Liang et al., 2019). These FINs not only provide valuable tools for ferroptosis studies, but also can be employed as potential therapeutic agents for cancer therapy. The detailed mechanisms of action and applications of these FINs are shown in Table 1.

\section{FERROPTOSIS AND RT}

Excessive ROS generated by RT through radiolysis of cellular water can damage biomolecules, including lipids, and therefore can be potentially linked to lipid peroxidation and ferroptosis. Previous studies suggested that IR can generate hydroxyl radicals and promote lipid peroxidation in lipid bilayers (Walden and Hughes; Shadyro et al., 2002). It was recently established by us and others that $\mathrm{RT}$ can trigger potent ferroptosis and that ferroptosis represents a critical part of RT-mediated tumor suppression (Lang et al., 2019; Lei et al., 2020; Ye et al., 2020). In this section, we summarize these recent findings on RT-induced ferroptosis, explore other potential mechanisms linking RT to ferroptosis, and further discuss the crosstalk between ferroptosis and other RT-induced cellular effects.

\section{The role and known mechanisms of RT-induced ferroptosis}

Substantial genetic and biochemical evidence forges a tight link between RT and ferroptosis in several cancers, including lung cancer, breast cancer, esophageal cancer, renal cell carcinoma, ovarian cancer, vulvar cancer, fibrosarcoma, and melanoma (Lang et al., 2019; Lei et al., 2020; Ye et al., 2020) (Fig. 2). First, RT is capable of significantly increasing the staining of C11-BODIPY and lipid peroxidation markers malondialdehyde (MDA) and 4-hydroxynonenal (4-HNE) in cancer cells and tumor samples, indicating that RT induces lipid peroxidation. Likewise, irradiated cells exhibit the increased expression of ferroptosis marker gene prostaglandin-endoperoxide synthase 2 (PTGS2), as well as the morphologic feature of ferroptosis with shrunken mitochondria with enhanced membrane density. Ferroptosis inhibitors (ferrostatin-1 and liproxstatin-1) or iron chelator DFO can partially restore clonogenic cell survival following RT in a wide range of cancer cell lines; notably, the survival restoring effect by ferroptosis inhibitors is comparable to or even more pronounced than that by inhibitors of other RCDs such as apoptosis and necroptosis. To minimize damages to normal tissues, a high dose of IR is usually delivered through multiple low doses, which is called dose fractionation. Fractionation generally includes conventionally fractionation (such as 2 Gy once daily, 5 times/week), hypofractionation (such as 3 Gy once daily, 5 times/week), and hyperfractionation (such as $1.1 \mathrm{~Gy}$ twice daily, 5 times/week) (Withers, 1985; Williams et al., 2006). Notably, different RT doses and fractionation schedules result in differential levels of ferroptosis; specifically, lipid peroxidation and ferroptosis can be augmented with increasing doses in the single-fraction case, whereas the single fraction at $10 \mathrm{~Gy}$ induces more lipid peroxidation than three fractions with each fraction at $5 \mathrm{~Gy}$ (3 $\times 5$ Gy) (Lang et al., 2019), which could provide insights for further investigations of ferroptosis in hypofractionated (e.g., stereotactic body radiation therapy), conventionally fractionated, and hyperfractionated RT (Thariat et al., 2013).

Mechanistically, IR induces lipid peroxidation and ferroptosis likely through at least three parallel pathways (Lang et al., 2019; Lei et al., 2020; Ye et al., 2020) (Fig. 2). First, IR can induce lipid peroxidation through generating excessive ROS. Specifically, IR-generated ROS can abstract electrons from PUFAs, resulting in the formation of PUFA radicals (PUFA ${ }^{\circ}$ ). Subsequently, these unstable carbon-centered radicals can interact promptly with oxygen molecules to produce lipid peroxyl radicals (PUFA-OO'), which then abstract $\mathrm{H}^{*}$ from other molecules via the Fenton reaction and eventually generate lipid hydroperoxides (PUFA-OOH) (Shadyro et al., 2002; Azzam et al., 2012). In addition, IR upregulates ACSL4 expression to promote PUFA-PLs biosynthesis, although the exact underlying mechanism by 
Table 1. The identified FINs and ferroptosis promoters

\begin{tabular}{|c|c|c|c|c|c|}
\hline Classification & Compound & Mechanism & $\begin{array}{l}\text { In } \\
\text { vivo }\end{array}$ & Clinic & Radiosensitizer \\
\hline \multirow[t]{10}{*}{ Class I FINs } & Erastin & Inhibit SLC7A11 activity & & & $\sqrt{ }$ \\
\hline & PE & Inhibit SLC7A11 activity & $\sqrt{ }$ & & \\
\hline & IKE & Inhibit SLC7A11 activity & $\sqrt{ }$ & & $\sqrt{ }$ \\
\hline & SAS & Inhibit SLC7A11 activity & $\sqrt{ }$ & $\sqrt{ }$ & $\sqrt{ }$ \\
\hline & Sorafenib & Inhibit SLC7A11 activity & $\sqrt{ }$ & $\sqrt{ }$ & $\sqrt{ }$ \\
\hline & Cyst(e)inase & Cyst(e)ine depletion & $\sqrt{ }$ & & $\sqrt{ }$ \\
\hline & Glutamate & Inhibit SLC7A11 activity & & & \\
\hline & BSO & GSH depletion & $\sqrt{ }$ & & $\sqrt{ }$ \\
\hline & DPI2 & GSH depletion & & & \\
\hline & Cisplatin & GSH depletion & $\sqrt{ }$ & $\sqrt{ }$ & $\sqrt{ }$ \\
\hline \multirow[t]{5}{*}{ Class II FINs } & 1S,3R-RSL3 & Inhibit GPX4 activity & & & $\sqrt{ }$ \\
\hline & ML162 & Inhibit GPX4 activity & & & $\sqrt{ }$ \\
\hline & ML210 & Inhibit GPX4 activity & & & \\
\hline & Altretamine & Inhibit GPX4 activity & $\sqrt{ }$ & $\sqrt{ }$ & \\
\hline & Withaferin A & Inactivate/deplete GPX4 & $\sqrt{ }$ & & $\sqrt{ }$ \\
\hline \multirow[t]{2}{*}{ Class III FINs } & FIN56 & $\begin{array}{l}\text { Degrade GPX4, activate SQS and deplete } \\
\mathrm{CoQ}_{10}\end{array}$ & & & $\sqrt{ }$ \\
\hline & $\begin{array}{l}\text { Statins (fluvastatin, } \\
\text { simvas-tatin, lovastatin } \\
\text { acid) }\end{array}$ & $\begin{array}{l}\text { Inhibit HMG-CoA reductase (inhibit } \mathrm{CoQ}_{10} \\
\text { synthesis, reduce GPX4 expression) }\end{array}$ & $\sqrt{ }$ & $\sqrt{ }$ & $\sqrt{ }$ \\
\hline \multirow[t]{9}{*}{ Class IV FINs } & $\begin{array}{l}\text { Ferric ammonium } \\
\text { citrate/sulfate }\end{array}$ & Iron loading & & & \\
\hline & $\mathrm{FeCl}_{2}$ & Iron loading & $\sqrt{ }$ & & \\
\hline & Hemoglobin & Iron loading & $\sqrt{ }$ & $\sqrt{ }$ & $\sqrt{ }$ \\
\hline & Hemin & Iron loading & $\sqrt{ }$ & $\sqrt{ }$ & \\
\hline & Nonthermal plasma & Promote the release of $\mathrm{Fe}^{2+}$ from ferritin & $\sqrt{ }$ & & $\sqrt{ }$ \\
\hline & Lapatinib + siramesine & Upregulate TfR1 and downregulate FPN1 & $\sqrt{ }$ & $\sqrt{ }$ & $\sqrt{ }$ \\
\hline & Salinomycin & Inhibit iron translocation and deplete ferritin & $\sqrt{ }$ & $\sqrt{ }$ & $\sqrt{ }$ \\
\hline & Artesunate, DHA & $\begin{array}{l}\text { Endogenous } \mathrm{Fe}^{2+} \text { causes the cleavage of } \\
\text { endoperoxide bridge }\end{array}$ & $\sqrt{ }$ & $\sqrt{ }$ & $\sqrt{ }$ \\
\hline & FINO2 & $\begin{array}{l}\text { Inhibit GPX4 activity, Oxidize ferrous iron } \\
\text { and lipidome }\end{array}$ & & & \\
\hline \multirow{9}{*}{$\begin{array}{r}\text { Other FINs/ } \\
\text { promoters }\end{array}$} & BAY 87-2243 & Inhibit mitochondrial complex I & & & $\sqrt{ }$ \\
\hline & BAY 11-7085 & Upregulate HMOX1 & & & $\sqrt{ }$ \\
\hline & Auranofin/Ferroptocide & Inhibit thioredoxin & $\sqrt{ }$ & $\sqrt{ }$ & $\sqrt{ }$ \\
\hline & iFSP1 & Inhibit FSP1 & & & \\
\hline & $4-\mathrm{CBA}$ & $\mathrm{CoQ}_{10}$ depletion & $\sqrt{ }$ & & \\
\hline & DAHP & Inhibit GCH1 & $\sqrt{ }$ & & \\
\hline & Methotrexate & Inhibit DHFR & $\sqrt{ }$ & $\sqrt{ }$ & \\
\hline & MF-438/ CAY10566 & Inhibit SCD1 & $\sqrt{ }$ & & \\
\hline & JQ-1 & Promote ferritinophagy & $\sqrt{ }$ & & $\sqrt{ }$ \\
\hline
\end{tabular}


Table 1 continued

\begin{tabular}{|c|c|c|c|c|c|}
\hline Classification & Compound & Mechanism & $\begin{array}{l}\text { In } \\
\text { vivo }\end{array}$ & Clinic & Radiosensitizer \\
\hline \multirow[t]{8}{*}{ Nanoparticles } & AMSNs & GSH depletion & $\sqrt{ }$ & & \\
\hline & LDL-DHA & Loading natural omega 3 fatty acid & $\sqrt{ }$ & & \\
\hline & ZVI NPs & Iron loading & $\sqrt{ }$ & & \\
\hline & $\begin{array}{l}\text { FeGd-HN@Pt@LF/ } \\
\text { RGD2 }\end{array}$ & Increase intracellular $\mathrm{Fe}^{2+}$ and $\mathrm{H}_{2} \mathrm{O}_{2}$ levels & $\sqrt{ }$ & & \\
\hline & DGU:Fe/Dox & $\begin{array}{l}\text { delivery system releasing } \mathrm{Fe}^{3+} \text { and } \\
\text { doxorubicin }\end{array}$ & $\sqrt{ }$ & & \\
\hline & SRF@Fe"IA & $\begin{array}{l}\text { Consists of } \mathrm{Fe}^{3+} \text { ion, tannic acid and } \\
\text { sorafenib }\end{array}$ & $\sqrt{ }$ & & \\
\hline & PSAF NCs & Increase intracellular $\mathrm{Fe}^{2+}$ levels & $\sqrt{ }$ & & \\
\hline & MON-p53 & Iron loading, inhibit SLC7A11 & $\sqrt{ }$ & & \\
\hline
\end{tabular}

This table lists compounds currently known to induce or promote ferroptosis, including their classification, mechanism, suitability for in vivo administration, and availability as radiosensitizers.

which IR induces ACSL4 levels remains unclear (Lei et al., 2020). Consistently, IR exerts a pronounced effect on ferroptosis-associated lipid metabolism, with multiple LysoPLs and diacylglycerols (DAGs) reported to be significantly increased following irradiation (Ye et al., 2020). Increased levels of LysoPLs (Colles and Chisolm, 2000; Dixon et al., 2015; Yang et al., 2016; Kagan et al., 2017; Zhang et al., 2019a) and DAGs (Zhang et al., 2019a; Zou et al., 2019) have also been observed following the treatment with FINs, suggesting that IR and FIN treatment induce similar lipidomic signatures, which is in line with their shared effects to trigger ferroptosis. Finally, IR also leads to GSH depletion, which weakens GPX4-mediated ferroptosis defense and further promotes ferroptosis (Ye et al., 2020).

In one study, IR was shown to repress SLC7A11 expression in an ATM-dependent manner, and it was proposed that IR-mediated SLC7A11 repression triggers ferroptosis by reducing cystine uptake and $\mathrm{GSH}$ synthesis (Lang et al., 2019) (Fig. 2). However, other studies revealed that the expression of SLC7A11 is actually induced by IR, likely as an adaptive response (Xie et al., 2011; Lei et al., 2020). Although the mechanism underlying the upregulation of SLC7A11 upon IR remains undefined, it likely involves NRF2 and/or ATF4, both of which are generally activated by IR and are known to regulate SLC7A11 transcription (McDonald et al., 2010; Zong et al., 2017; Koppula et al., 2020). Therefore, it appears that IR can either activate or repress SLC7A11 expression in a context (cell line, IR dose or duration)-dependent manner. Taken together, the multifaceted evidence from different studies establishes a robust link between ferroptosis and RT, and suggests several underlying mechanisms for RT-induced ferroptosis.

\section{Other potential mechanisms}

Because multiple metabolic pathways are involved in the regulation of ferroptosis and several ferroptosis regulators are RT-responsive genes (Fig. 1), other potential mechanisms might also contribute to RT-induced ferroptosis, which will be further discussed in this subsection. As a central effector of RT, p53 is not only activated by IR (Fei and ElDeiry, 2003; Gudkov and Komarova, 2003), but also plays a dual role in the ferroptosis network (Kang et al., 2019) (Fig. 1). Specifically, p53 was shown to repress SLC7A11 transcription by binding directly to the p53 response element in the SLC7A11 promoter region or by interacting with ubiquitin-specific protease 7 (USP7) to reduce the levels of H2B monoubiquitination on the SLC7A11 gene regulatory region, thereby exerting a pro-ferroptosis effect in response to oxidative stress (Jiang et al., 2015; Wang et al., 2019c). It was further shown that p53-mediated SLC7A11 repression promotes ferroptosis in an ALOX12-dependent manner (Chu et al., 2019). In addition, p53 can induce the expression of spermidine/spermine N1-acetyltransferase 1 (SAT1) to upregulate ALOX15, thus promoting ferroptosis upon ROS stress (Ou et al., 2016). p53 regulation of glutaminases 2 (GLS2) (Hu et al., 2010; Suzuki et al., 2010; Gao et al., 2015) or ferredoxin reductase (FDXR) (Hwang et al., 2001; Zhang et al., 2017) might also potentially contribute to ferroptosis. In contrast, other studies showed that p53 can function as a ferroptosis inhibitor by upregulating p21 to maintain the GSH levels upon metabolic stress (Tarangelo et al., 2018), or by blocking dipeptidyl-peptidase-4 (DPP4) activity in a transcription-independent manner (Xie et al., 2017). Given the context dependent role of p53 in governing ferroptosis, 


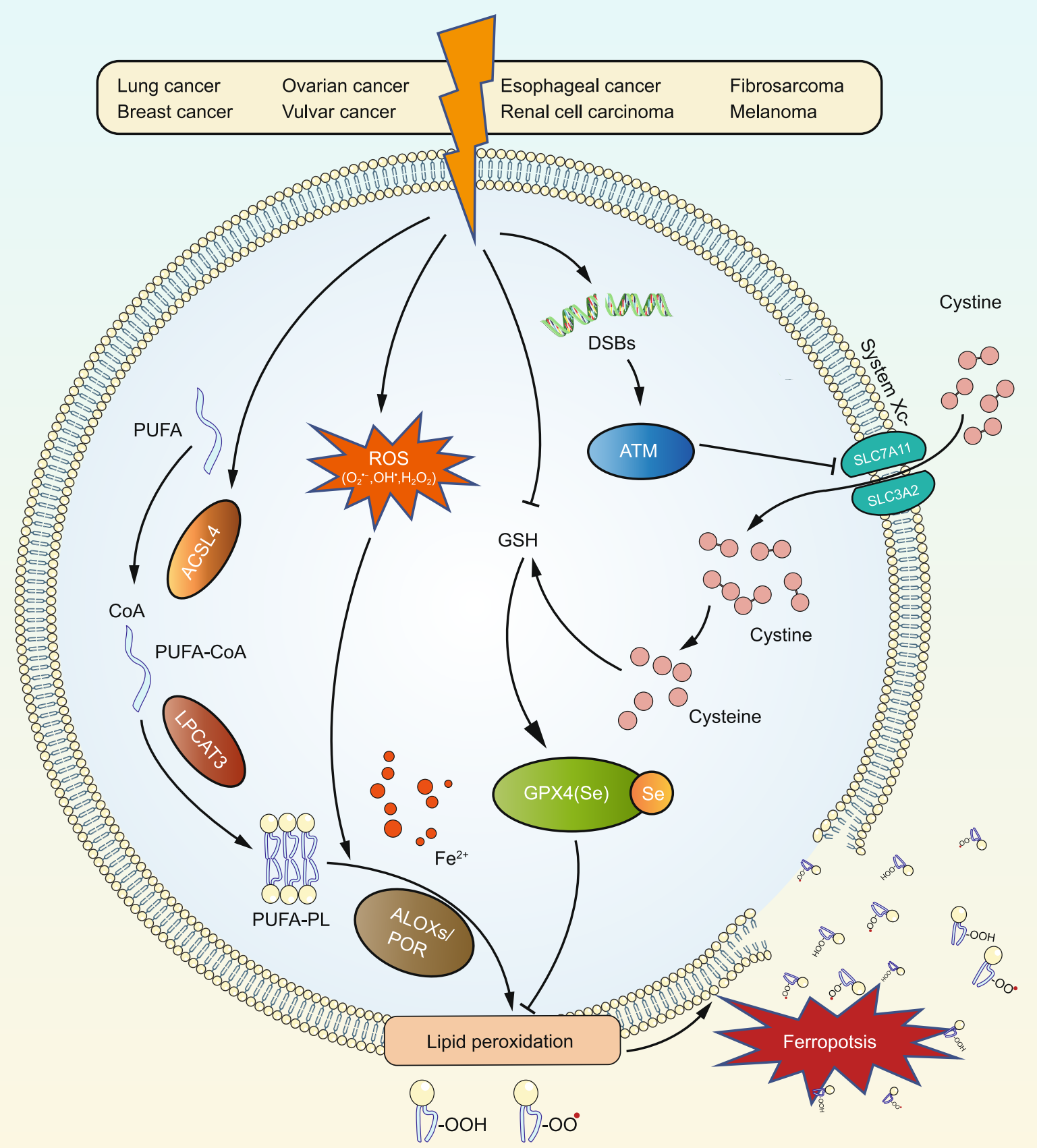

Figure 2. Mechanisms of radiotherapy-induced ferroptosis. Radiotherapy (RT) has been revealed to induce ferroptosis in the indicated cancers through several parallel pathways. RT-induced ROS in concert with RT-induced ACSL4 expression trigger PUFAPL peroxidation and ferroptosis. RT also depletes GSH, dampens GPX4-mediated ferroptosis defense, thereby promoting ferroptosis. In addition, RT can repress SLC7A11 expression in an ATM-dependent manner to further promote ferroptosis or upregulate SLC7A11 expression as an adaptive response for ferroptosis protection, depending on the context.

whether RT-induced p53 activation contributes to or antagonizes RT-induced ferroptosis merits further investigations.

Another signaling node that potentially links RT to ferroptosis is AMPK. RT has been widely demonstrated to activate AMPK (Sanli et al., 2010; Sanli et al., 2014). Interestingly, AMPK activation also appears to exert context dependent effects on ferroptosis (Fig. 1). AMPK-mediated phosphorylation of beclin-1 was reported to inhibit system $x_{c}^{-}$ 
activity, thereby promoting ferroptosis (Song et al., 2018), while energy stress-induced AMPK activation was recently shown to inhibit ferroptosis by restraining PUFA-PL biosynthesis (Lee et al., 2020; Li et al., 2020). The exact role of AMPK in RT-induced ferroptosis therefore remains to be examined.

It is known that RT induces the expression of MDM2 in an ATM- or p53-dependent manner (Chen et al., 1994; Maya et al., 2001). Recently MDM2 was shown to promote ferroptosis through regulating lipid metabolism and FSP1 expression (Venkatesh et al., 2020), suggesting a possible role of MDM2 in RT-induced ferroptosis (Fig. 1). As discussed in a previous section, The $\mathrm{GCH} 1-\mathrm{BH}_{4}$ signaling axis constitutes a GPX4-independent ferroptosis defense system. It was observed that IR decreased the level and bioavailability of $\mathrm{BH}_{4}$ in vivo, presumably because IR induces the expression of $\mathrm{GCH} 1$ feedback regulatory protein (GFRP), thereby potentiating GFRP-mediated inhibition of GCH1 activity (Li et al., 2010; Berbee et al., 2011; Cheema et al., 2014; Pathak et al., 2014) (Fig. 1). This raises the possibility that $\mathrm{GCH} 1$ might also be involved in regulating IRinduced ferroptosis. Furthermore, IR promotes iron release from heme by inducing heme oxygenase-1 (HO-1) or from ferritin (Han et al., 2005; Wolszczak and Gajda, 2010; Hassannia et al., 2018) (Fig. 1). However, it has also been reported that IR upregulates the expression of FTH1 (Choudhary et al., 2020), which plays an important role in reducing oxidative stress and promoting radioresistance (Pang et al., 2016). Therefore, it remains obscure whether RT promotes ferroptosis by regulating iron metabolism.

Finally, transmission electron microscopy revealed that mitochondria exhibit ferroptotic cell features following IR, implying that mitochondria are potentially involved in IR-induced ferroptosis (Lei et al., 2020). Indeed, IR has been shown to dramatically alter mitochondrial structure or function, including mitochondrial DNA, mitochondrial permeability, ETC activity, oxidative phosphorylation, and mitochondrial antioxidant enzyme function, resulting in extensive mitochondrial ROS production (Leach et al., 2001; Kam and Banati, 2013) (Fig. 1). Further studies are required to determine the potential role of mitochondria in IR-induced ferroptosis.

Collectively, diverse signaling nodes and cellular processes have been linked to both RT and ferroptosis; therefore, RT-induced ferroptosis likely involve a multitude of mechanisms. Future investigations are needed to develop a comprehensive molecular understanding of RT-induced ferroptosis.

\section{Crosstalk between ferroptosis and other RT-induced cellular effects}

As introduced in a preceding section, one major cellular effect triggered by RT is to induce DNA damage in the nucleus. Ferroptosis, on the other hand, is triggered by lipid damage, namely lipid peroxidation, caused by toxic accumulation of lipid hydroperoxides on cellular membranes. This raised the question of whether there exists any interaction between ferroptosis and DNA damage upon RT. Recent studies revealed that neither perturbation of IR-induced ferroptosis by ferroptosis inhibitors nor augmentation of IR-induced ferroptosis by FINs affects IR-mediated DSBs (Lei et al., 2020; Ye et al., 2020). Further, microbeam radiation analysis showed that IR specifically targeting the nucleus induced phosphorylated $\mathrm{H} 2 \mathrm{~A}$ histone family member $\mathrm{X}$ ( $\mathrm{HH} 2 \mathrm{AX}$, a DSB marker) but did not produce 4-HNE (a lipid peroxidation marker), whereas levels of 4-HNE, but not those of $\mathrm{YH} 2 \mathrm{AX}$, were elevated following IR specifically targeting the cytoplasm, suggesting that ferroptosis induction in the cytoplasm and DNA damage in the nucleus can be uncoupled following IR (Ye et al., 2020).

However, from a signaling perspective, there seems to exist a crosstalk between DNA damage response and ferroptosis. IR induces DNA damage and thus activates ATM, p53, or RB (Sabin and Anderson, 2011; Maier et al., 2016), which can be linked to RT-induced ferroptosis and other types of RCD, including apoptosis, necroptosis and autophagy, collectively known as immunogenic cell death (ICD) (Kang and Tang, 2016) (Fig. 3A). These ICDs, together with RT-induced senescence-associated secretory phenotype (SASP), activate $\mathrm{T}$ cells and recruit them into tumor sites (Rao and Jackson, 2016; Herrera et al., 2017; Li et al., 2018), whereas interferon gamma (IFNy) secreted from $\mathrm{CD}^{+} \mathrm{T}$ cells further promotes RT-induced ferroptosis (Lang et al., 2019; Wang et al., 2019b) (Fig. 3A). Moreover, RTinduced autophagy can potentially promote ferroptosis through ferritinophagy, lipophagy, clockophagy and/or chaperone-mediated autophagy (Liu et al., 2020a) (Fig. 3A). Therefore, the immune system and autophagy may be involved in the intersection between RT-induced DNA damage and ferroptosis.

On the molecular level, multiple regulators possibly underlie the ATM- or p53-mediated crosstalk between ferroptosis and other types of RCD upon IR-induced DSBs (Fig. 3B). IR-induced activation of ATM mediates the downregulation of SLC7A11 expression, thereby contributing to IR-induced ferroptosis (Lang et al., 2019); ATM activation can also promote ferroptosis through the metal regulatory transcription factor 1 (MTF1)-Ferritin/FPN1 axis (Chen et al., 2020) (Fig. 3B). p53 can also be linked to the crosstalk between senescence and ferroptosis. As discussed earlier, p53 transcriptional target p21 suppresses ferroptosis by maintaining GSH levels (Tarangelo et al., 2018); p21 also promotes senescence in cells with irreparable DNA damage (Georgakilas et al., 2017) (Fig. 3B). AMPK is activated in an ATM-dependent manner to mediate multiple cellular effects upon IR-induced DSBs (Sanli et al., 2014). Besides regulating p21-mediated senescence, AMPK is at least partially responsible for IR-induced autophagy by rescinding mammalian target of rapamycin (mTOR)-mediated autophagy inhibition or promoting beclin1-mediated autophagy (Sanli 

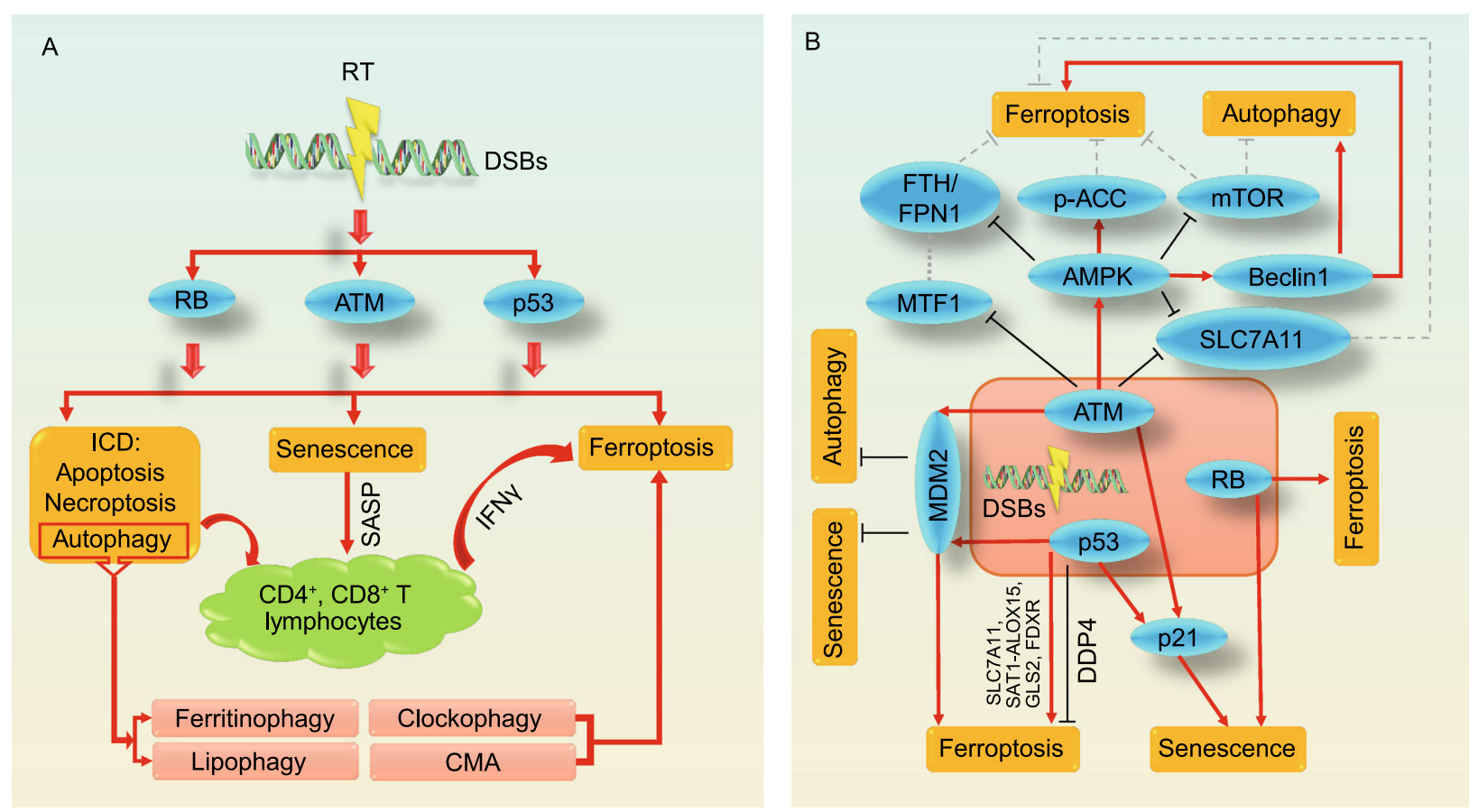

Figure 3. The crosstalk among RT-induced DSBs, immune system activation and ferroptosis. (A) Radiotherapy (RT) induces DSBs and thus activates ATM, p53 and RB, promoting senescence, apoptosis, necroptosis, autophagy, and ferroptosis. Immunogenic cell deaths (ICDs; including apoptosis, necroptosis, and autophagy), together with RT-induced senescence-associated secretory phenotype (SASP), contribute to T cell activation, which secretes IFNy to further promote RT-induced ferroptosis. Additionally, RT-induced autophagy may modulate ferroptosis through ferritinophagy, lipophagy, clockophagy or chaperone-mediated autophagy (CMA). (B) Specific crosstalk mechanisms between ferroptosis and other forms of regulated cell death under RT-induced DSBs, in which ATM, p53 and RB play central roles.

et al., 2014; Zhang et al., 2016); on the other hand, mTOR, beclin1, and AMPK-mediated ACC phosphorylation can modulate ferroprosis, positively or negatively (Song et al., 2018; Lee et al., 2020; Yi et al., 2020) (Fig. 3B). In addition, MDM2 can be up-regulated by IR in an ATM- or p53-dependent fashion (Chen et al., 1994; Maya et al., 2001); subsequently, MDM2 promotes ferroptosis (Venkatesh et al., 2020), but suppresses autophagy and senescence (Wu and Prives, 2018; Liu et al., 2019a; Liu et al., 2020b) (Fig. 3B). Another crosstalk between senescence and ferroptosis is the RB protein, which mediates cellular senescence upon IR and also potentiates ferroptosis (Sabin and Anderson, 2011; Louandre et al., 2015) (Fig. 3B).

In summary, multiple lines of evidence suggest that RTinduced ferroptosis does not affect DNA damage, whereas RT-induced DNA damage appears to affect ferroptosis through diverse mechanisms. There also exist multiple layers of crosstalk between ferroptosis and other RT-induced cellular effects. Further characterization of these interactions may yield new insights into the mechanisms of radioresistance and strategies for radiosensitization, which will be further discussed in the next section.

\section{THERAPEUTIC POTENTIAL OF FERROPTOSIS IN RT-MEDIATED TUMOR SUPPRESSION}

$\mathrm{RT}$ destroys tumors precisely in localized areas by IR with a high objective response rate (ORR) and activates the immune system to attack target lesions and distant metastases by inducing ICD (Thariat et al., 2013; Herrera et al., 2017). However, intrinsic or acquired radioresistance is a long-standing challenge in RT; as such, RT is generally combined with other therapies, including chemotherapy, targeted therapy, and immunotherapy, to improve the radiosensitivity and to eliminate potential cancer cells outside the radiation field. In view of this, the landing points for investigating the therapeutic relevance of ferroptosis in RT include: 1) whether ferroptosis and its regulators modulate radiosensitivity, 2) whether targeting ferroptosis contributes to radiosensitization, and 3) how to further incorporate immunotherapy into targeting ferroptosis in RT. Our following discussion in this section will center on these questions.

\section{Ferroptosis-mediated radiosensitization}

Pharmacological blockade of ferroptosis was shown to protect cancer cells from RT, and RT induced less potent lipid 
A

Radioresistance: KEAP1 mutation,

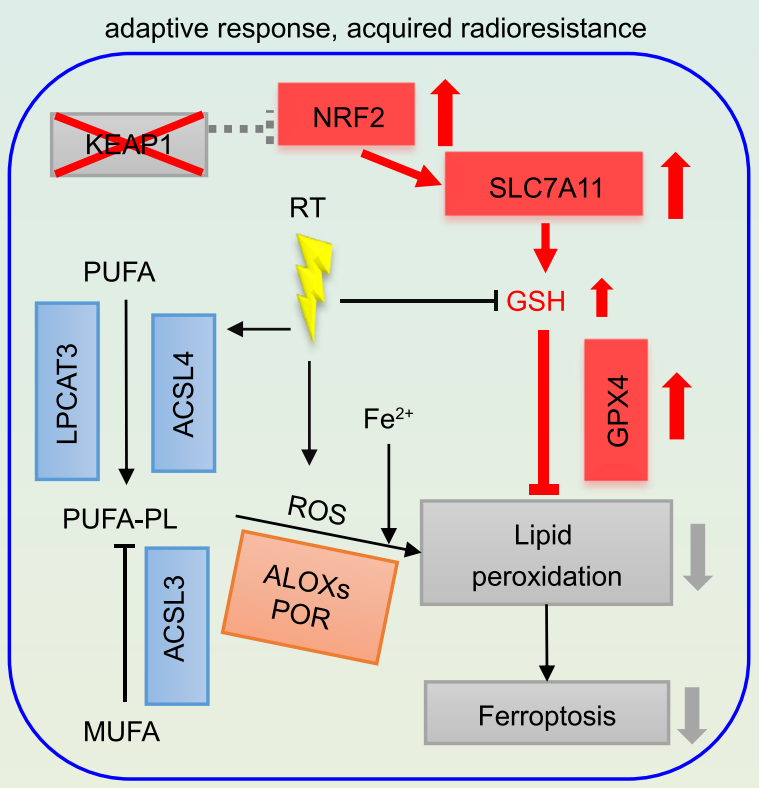

C

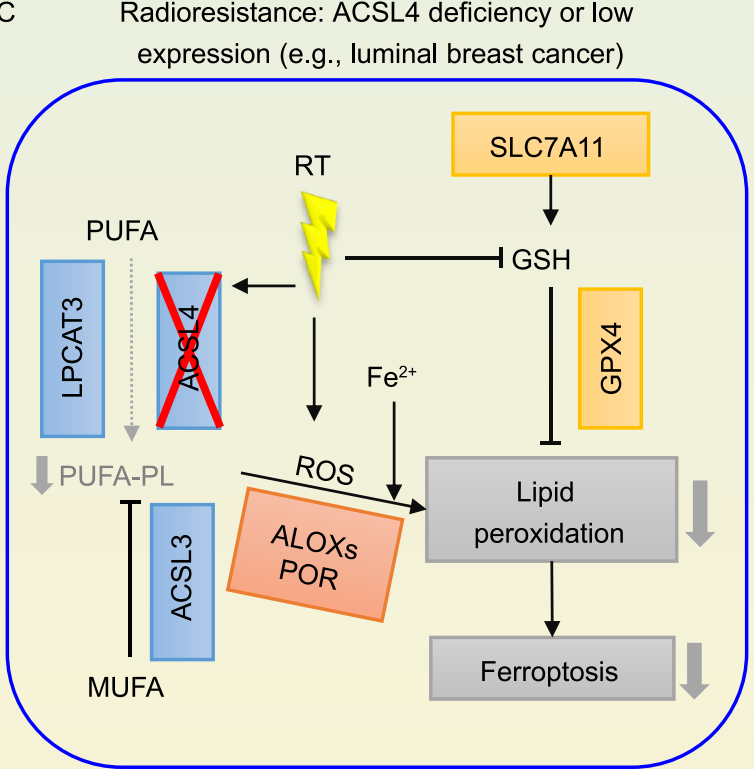

B

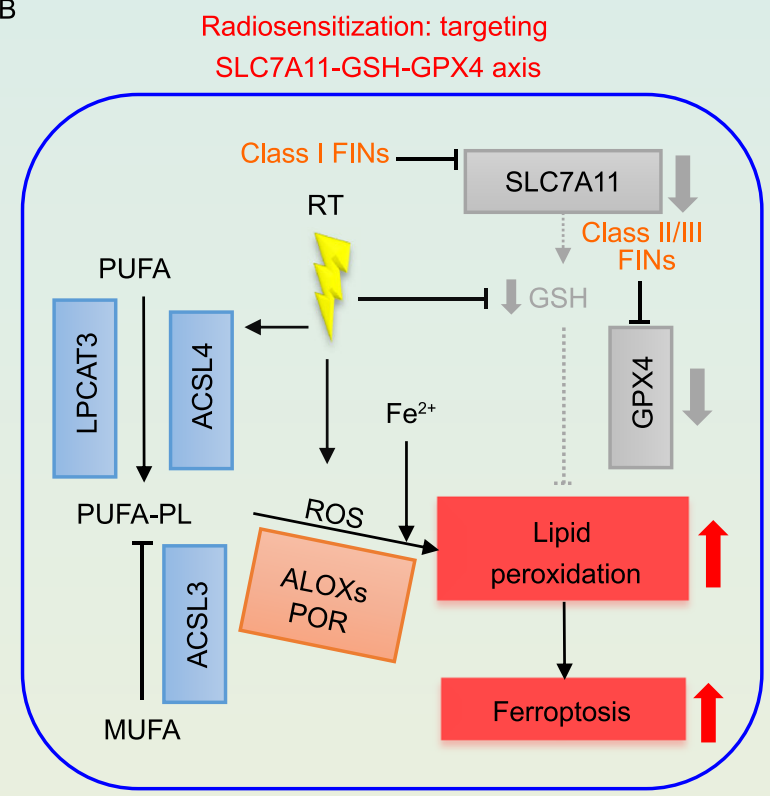

D

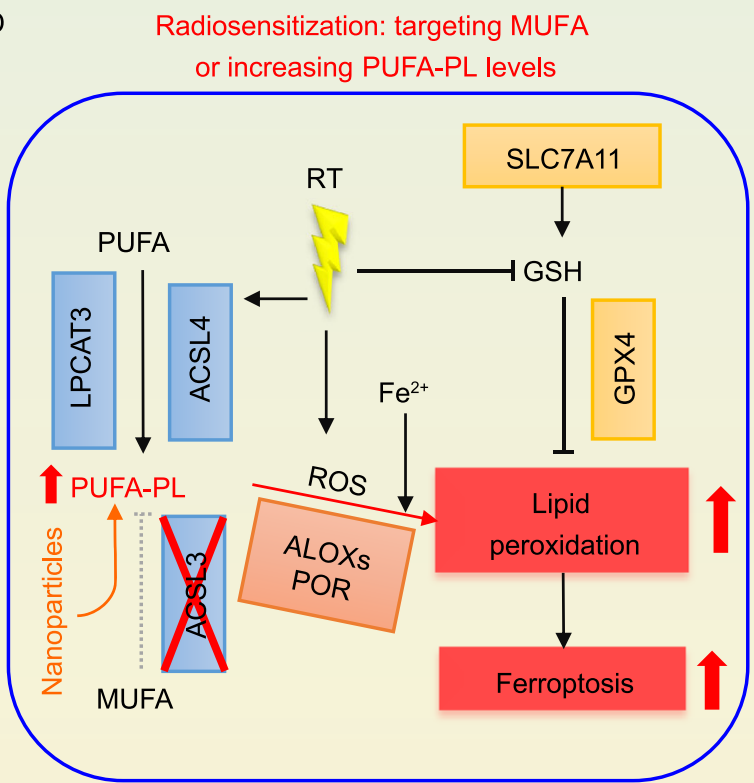

Figure 4. Radioresistance mechanisms due to ferroptosis inactivation and radiosensitization strategies by inducing ferroptosis. (A) Radiotherapy (RT) induces the expression of SLC7A11 or GPX4 as an adaptive response to protect cancer cells from ferroptosis, thereby compromising RT-induced cell death and possibly contributing to acquire radioresistance. In addition, KEAP1 mutant cancer cells are radioresistant due to ferroptosis resistance partly caused by high SLC7A11 expression. (B) Class I FINs targeting SLC7A11 or class II/III FINs targeting GPX4 potentiate RT-induced lipid peroxidation and ferroptosis, thereby sensitizing cancer cells to RT. (C) ACSL4 deficiency or low expression (such as in luminal breast cancer) inhibits RT-induced ferroptosis by blocking PUFA-PL synthesis, resulting in radioresistance. (D) Inhibiting ACSL3-mediated MUFA-PL synthesis or increasing PUFA-PL levels via nanoparticles promotes RT-induced ferroptosis, thereby sensitizing cancer cells to RT. 
peroxidation and ferroptosis in FIN-resistant cancer cells, which also appear to be radioresistant (Lang et al., 2019; Lei et al., 2020; Ye et al., 2020). Several studies uncovered that IR induces the expression of SLC7A11 and GPX4 as an adaptive response to protect cells from ferroptosis, contributing to radioresistance (Fig. 4A); consequently, depletion or inhibition of SLC7A11 (or GPX4) enables significant radiosensitization by boosting IR-induced ferroptosis (Xie et al., 2011; Pan et al., 2019; Lei et al., 2020) (Fig. 4B). Likewise, deficiency of the tumor suppressor KEAP1 (which is frequently mutated in lung cancer) inhibited IR-induced ferroptosis at least partly through stabilizing NRF2 and upregulating SLC7A11, leading to radioresistance (Lei et al., 2020) (Fig. 4A). Further, inactivation of ACSL4 impaired the biosynthesis of PUFA-PLs, thereby inhibiting IR-induced ferroptosis and causing radioresistance (Lang et al., 2019; Lei et al., 2020) (Fig. 4C), whereas ablation of ACSL3 diminishes the biosynthesis of MUFA-PLs (which suppress ferroptosis (Magtanong et al., 2019)), leading to enhanced IR-induced ferroptosis and radiosensitization in cancer cells (Lang et al., 2019) (Fig. 4D). Whether other ferroptosis regulators may also modulate radiosensitivity requires further investigation.

Many tumors exhibit at least somewhat susceptibilities to ferroptosis, and tumor sensitivities to RT among different tumor types do not appear to strictly correlate with their susceptibilities to ferroptosis. For example, tumor types in which RT is an important treatment modality, such as hepatocellular carcinoma, pancreatic cancer, diffuse large B cell lymphoma, and triple-negative breast cancer, as well as radioresistant tumor types, including renal cell carcinoma and ovarian cancer, are all somewhat sensitive to ferroptosis (Zou and Schreiber, 2020). This susceptibility may be associated with the high dependence of some tumors, such as a portion of pancreatic cancer and renal cell carcinoma, on cystine uptake. Intriguingly, certain treatment-resistant cancer cells, such as therapy-resistant mesenchymal cancer cells or drug-tolerant persister cancer cells, are vulnerable to ferroptosis, likely because certain unique state of these cancer cells somehow renders them to be particularly dependent on GPX4 function (Hangauer et al., 2017; Viswanathan et al., 2017); likewise, melanoma cells undergoing dedifferentiation upon BRAF inhibitor treatment also exhibit an increased susceptibility to ferroptosis (Tsoi et al., 2018). It was further shown that withaferin A-induced ferroptosis suppresses tumor growth and recurrence in therapy-resistant high-risk neuroblastoma (Hassannia et al., 2018). Further investigations of tumor susceptibility to ferroptosis will help guide strategies to augment RT in cancer treatment.

The hypoxic tumor microenvironment represents an important mechanism of radioresistance (Fig. 5A), which is likely attributed to the "oxygen fixation hypothesis" and hypoxia-inducible factors (HIFs) activation (Wang et al., 2019a). On the other hand, hypoxia promotes ROS production (Fig. 5A); consequently, hypoxic tumor cells strongly rely on antioxidant systems to maintain redox homeostasis, and GSH inhibition was shown to overcome hypoxia-mediated radioresistance (Bump and Brown, 1990; Wang et al., 2019a). As discussed above, ROS contributes to POR-mediated lipid peroxidation and ferroptosis(Yan et al., 2020) (Fig. 5A). Intriguingly, HIFs (HIF-1 and -2) have been reported to confer susceptibility to ferroptosis (Fig. 5A). Mechanistically, HIF-2a activates hypoxia-induced, lipid droplet-associated protein (HILPDA) to promote the formation of PUFA-PLs and thereby increase the susceptibility of cancer cells to ferroptosis (Singhal et al., 2019; Zou et al., 2019). HIF-1a activation also sensitizes renal cancer cells to ferroptosis (Zou et al., 2019). In brief summary, while it is well established that hypoxia promotes radioresistance, hypoxiainduced ROS and HIF activation appear to promote ferroptosis (Fig. 5A). More studies are required to clarify the likelihood and specific mechanisms by which ferroptosis induction can reduce radioresistance of hypoxic cancer cells in different cancer settings (Fig. 5B).

In contrast, some drug-resistance cancers that may only respond to RT treatment develop the ability to evade ferroptosis (Boumahdi and de Sauvage, 2020). For example, luminal breast cancer is generally associated with low expression of ACSL4 (Doll et al., 2017), and some breast cancer cells upregulate prominin2 expression to promote iron excretion in the form of ferritin, rendering such cells resistant to ferroptosis (Brown et al., 2019). KRAS mutant lung cancers frequently overexpress ACSL3 and thus are equipped to synthesize more MUFA-PLs to protect against ferroptosis (Padanad et al., 2016), while a portion of lung adenocarcinoma cells exhibit high expression of iron-sulfur cluster biosynthesis enzyme cysteine desulfurase (NFS1), thus limiting the reactive iron available for ferroptosis by storing iron in iron-responsive proteins (Alvarez et al., 2017). In addition, anaplastic lymphoma kinase (ALK) positive lymphoma cells can shift the cholesterol synthesis to squalene formation, thereby counteracting lipid peroxidation and ferroptosis (Garcia-Bermudez et al., 2019). A common barrier for cancer therapy is the NRF2 activation in response to therapeutic stress (de la Vega et al., 2018), which also serves as a potential crosstalk between radioresistance and ferroptosis resistance. Overall, ferroptosis plays an important role in RT-mediated tumor suppression, and therefore inducing ferroptosis in RT-resistant tumors represents a promising strategy for radiosensitization. However, for ferroptosis-resistant tumors, which are also likely to be radioresistant, how to minimize their dual resistance remains to be further studied.

\section{Combining RT with FINs for tumor radiosensitization}

As discussed above, genetic perturbation of the anti-ferroptosis systems promotes radiosensitization in diverse cancer cells. Preclinical analyses by several recent studies also showed that FINs can synergize with RT in cancer treatment (Lang et al., 2019; Lei et al., 2020; Ye et al., 2020). For example, class I FINs targeting SLC7A11, such as erastin 


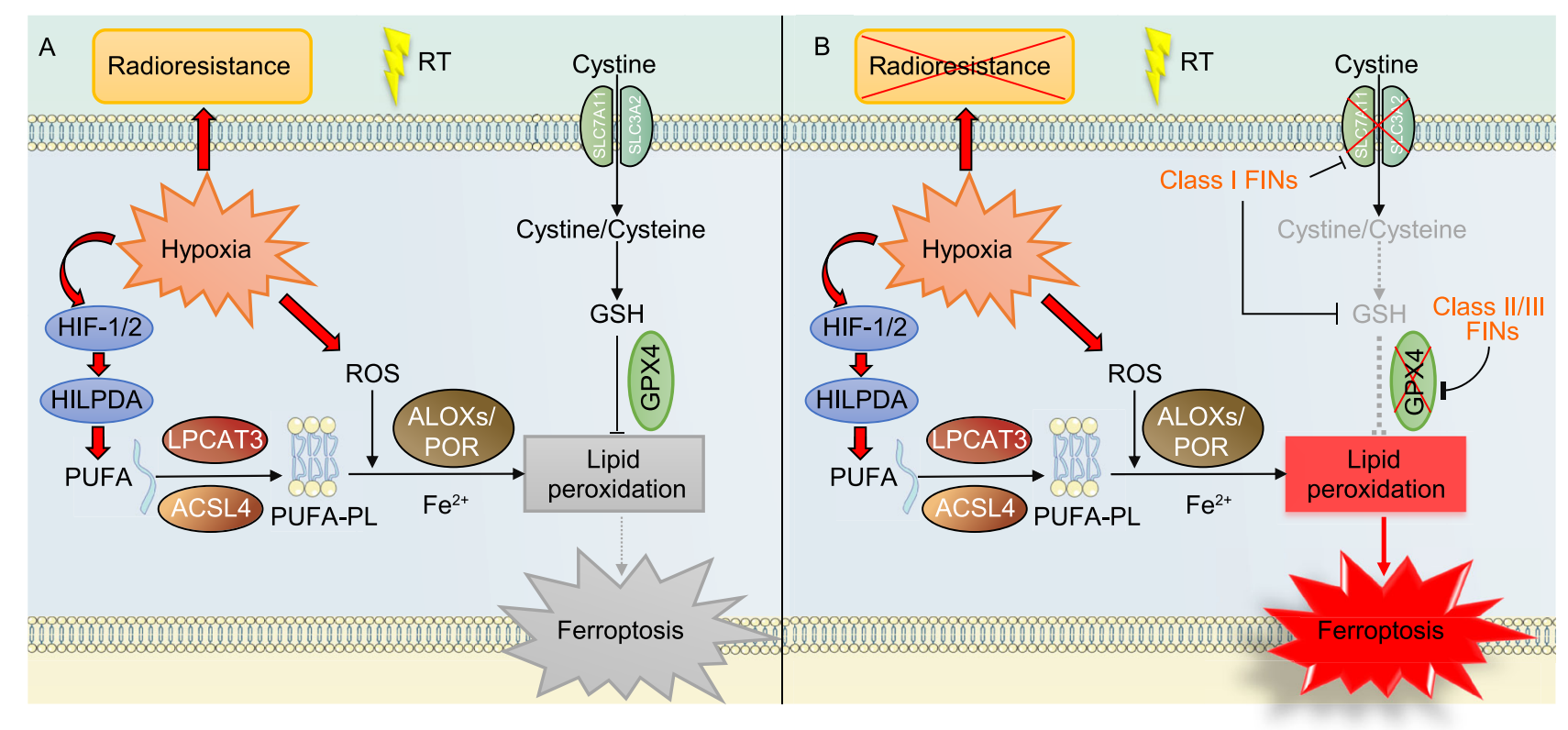

Figure 5. Interactions of hypoxia with ferroptosis and radioresistance, and potential strategies targeting ferroptosis to overcome hypoxia-induced radioresistance. (A) Hypoxia causes radioresistance possibly through "oxygen fixation hypothesis" and HIF activation. Hypoxia also induces the levels of ROS and HIF1/2, which have been shown to promote lipid peroxidation. Therefore, this regulation can potentially increase the susceptibility of cancer cells to ferroptosis. However, hypoxia might also upregulate ferroptosis defense systems (e.g., the SLC7A11-GSH-GPX4 axis) to counteract ferroptosis. (B) By inhibiting ferroptosis defense systems, FINs promote RT-induced ferroptosis and might overcome the radioresistance caused by hypoxia.

and sulfasalazine (SAS), class II FINs targeting GPX4, such as RSL3 and ML162, and class III FINs depleting CoQ and GPX4, such as FIN56, could all sensitize non-small cell lung cancer cells to RT in vitro (Lei et al., 2020) (Fig. 4B). Further, SAS (an FDA approved drug that is capable of inhibiting SLC7A11) was shown to exhibit a significant radiosensitizing effect in both cell line-derived xenografts (CDXs) and patientderived xenografts (PDXs) of ovarian cancer and KEAP1 mutant lung cancer; importantly, ferroptosis inhibitor treatment confirmed that SAS-mediated radiosensitization was indeed mediated by ferroptosis induction (Lang et al., 2019; Lei et al., 2020). Likewise, it was demonstrated that cyst(e) inase (which degrades extracellular cystine and cysteine and therefore operates similar to class I FINs), could sensitize cancer cells or tumors to RT (Lang et al., 2019). Another study showed that RT in combination with imidazole ketone erastin (IKE) or sorafenib (both of which are class I FINs inhibiting SLC7A11 activity) caused dramatic tumor suppression in both CDXs and PDXs (Ye et al., 2020) (Fig. 4B). In all of these studies, the combination of class I FINs with $\mathrm{RT}$ appeared to be well tolerated in vivo. Collectively, these studies suggest that administration of compounds targeting SLC7A11 to promote RT-induced ferroptosis is likely a promising strategy for radiosensitization in vivo, which is in line with other studies targeting SLC7A11 for tumor suppression (Badgley et al., 2020; Hu et al., 2020). Moreover, S/c7a11 deficient mice are viable with no overt phenotype (Sato et al., 2005; McCullagh and Featherstone, 2014), further indicating the safety of SLC7A11 inhibitors.

Inhibiting GSH synthesis or targeting GPX4 could provide an alternative approach for radiosensitization, especially considering that certain drug-resistant cancer cells are highly dependent on GPX4 for survival (Hangauer et al., 2017) (Fig. 4B). Inhibition of GSH synthesis by buthionine sulphoximine (BSO) to sensitize cancer cells to RT has been well established (Bump and Brown, 1990). Recent studies also showed that RSL3, ML162, and FIN56 have potent radiosensitizing effects in vitro (Lang et al., 2019; Lei et al., 2020; Ye et al., 2020); however, these drugs are not suitable for in vivo treatment due to their suboptimal pharmacokinetics (Hangauer et al., 2017). In this regard, withaferin A and altretamine (FDA-approved drugs for cancer therapy), with function to inhibit GPX4, exhibited favorable anti-tumor activity in animal models, representing another option for targeting GPX4 in vivo (Woo et al., 2015; Hassannia et al., 2018). It should be noted that Gpx4 knockout mice are embryonic lethal, thereby raising concerns on potential toxicity issues of GPX4 inhibitors for in vivo treatment (Yoo et al., 2012; Angeli et al., 2014); however, some cancers appear to be more sensitive to GPX4 inhibitors compared to 
their corresponding normal cells (Zou et al., 2019), suggesting that there might exist a therapeutic window for targeting GPX4 in certain cancers. Further studies are required to define the therapeutic window of GPX4 inhibition in cancer treatment and to explore techniques to target GPX4 locally in tumors for radiosensitization.

\section{The relevance of ferroptosis to RT combined} with immunotherapy

Immune system activation is an integral part of RT-mediated anticancer effects. On one hand, RT induces ICDs to expose tumor antigens and to activate antigen presenting cells (e.g., dendritic cells), promoting the migration of dendritic cells to the draining lymph nodes, leading to $T$ cell initiation in the lymph nodes and subsequent infiltration of $\mathrm{CD}^{+} \mathrm{T}$ cells into the irradiated field or unirradiated distant tumor sites. On the other hand, RT reprograms the tumor microenvironment to favor the recruitment and functioning of effector $T$ cells, making tumor cells more susceptible to T cell attack (Herrera et al., 2017). However, RT also upregulates the expression of programmed death-ligand 1 (PD-L1), a major checkpoint protein in the tumor immunosuppressive microenvironment, which assists cancer cells to escape from T-cell attack (Kordbacheh et al., 2018).

Notably, recent studies identified ferroptosis as a novel intersection between immunotherapy and RT (Lang et al., 2019; Wang et al., 2019b). It was shown that activated $\mathrm{CD}^{+}$ $T$ cells secrete IFNy during immunotherapy, which downregulates the expression of SLC7A11 (as well as its regulatory partner SLC3A2) and subsequently inhibits cystine uptake in cancer cells, thereby augmenting lipid peroxidation and ferroptosis. The combination of immune checkpoint inhibitors (ICls) with cyst(e)inase potentiated tumor ferroptosis and $\mathrm{T}$ cell-mediated antitumor immune responses in vivo. SLC7A11 expression in tumors was found to negatively correlate with $\mathrm{CD}^{+} \mathrm{T}$ cell counts and IFNy expression in tumors, and prognosis of cancer patients (Wang et al., 2019b). Further, IFNy secreted by $\mathrm{CD}^{+} \mathrm{T}$ cells was shown to promote RT-induced ferroptosis, which is likely caused by the synergistic repression of SLC7A11 expression by RT and IFNy. ICls, including PD-L1 or cytotoxic T-lymphocyte-associated protein 4 (CTLA-4) antibodies, in combination with RT synergistically induced tumor ferroptosis, while blocking ferroptosis, pharmacologically or genetically, attenuated the therapeutic effectiveness afforded by combining $\mathrm{ICls}$ with $\mathrm{RT}$; conversely, the therapeutic synergy of immunotherapy and RT could be further enhanced by inactivating SLC7A11 in tumors (Lang et al., 2019).

RT is commonly administered in combination with immunotherapy, particularly ICls, but this combination therapy seem to lack the expected survival benefits in certain tumors (Malhotra et al., 2017), highlighting an urgent need to identify specific biomarkers to define which tumors could be sensitive to the combination therapy. In this regard, tumors with low expression of anti-ferroptosis genes (such as SLC7A11) and/or high expression of pro-ferroptosis genes (such as ACSL4) could indicate that such tumors are particularly susceptible to ferroptosis and therefore might be suitable for this combination therapy. For those tumors that exhibit ferroptosis resistance features (such as with high expression of anti-ferroptosis genes and/or low expression of pro-ferroptosis genes), combining FINs with immunotherapy and RT might be a good strategy to enhance tumor ferroptosis and sensitize such tumors to immunotherapy and RT; however, whether this triple therapy strategy will also increase toxicity in normal tissues remains to be determined.

\section{CONCLUSIONS AND FUTURE PERSPECTIVES}

Recent studies establish a critical role of ferroptosis in RT and further suggest therapeutic strategies to target ferroptosis in RT as well as immunotherapy. Below we highlight a few key questions for further translating these findings into clinical applications. First, there exist significant differences in radiosensitivity among different types of cancer; even within the same tumor type, radiosensitivity might vary considerably among individuals due to tumor heterogeneity. Therefore, identifying suitable biomarkers for individualized RT has been an unmet need in RT research. In this regard, elevated levels of ferroptosis marker 4-HNE were found in tumor samples from patients treated with RT compared with matched tumor samples before RT; importantly, patients with strongly-positive levels of 4-HNE appeared to have better RT response and longer survival than those with weak/moderate levels of 4-HNE, suggesting an important role of ferroptosis in patients receiving RT (Lei et al., 2020). Therefore, further dissecting the interaction between RT and ferroptosis and using this information to develop mechanism-based biomarkers for patient stratification may help identify radiosensitive individuals and define populations suitable for co-treatment with FINs.

Further, although FIN + RT combination therapies appeared to be safe in preclinical studies, other studies demonstrated that ferroptosis might also be involved in RTinduced normal tissue damage, such as RT-induced lung injury (Li et al., 2019a; Li et al., 2019b). Therefore, it will be important to further clarify whether RT combination with FIN causes less toxicities to normal tissues than tumors (i.e., whether there exists an optimal therapeutic window). The development of nanomaterials with FIN activity may be an alternative way to address this issue.

Finally, current studies investigating the role of ferroptosis in RT have focused on X-rays, a type of photon with low LET. With the continuous development of RT physics, proton therapy and other high LET radiations, such as carbon ions, have been developed in recent years, and some of them are shown to achieve superior therapeutic efficacies than photon therapy (Mohan and Grosshans, 2017; Mohamad et al., 2019). Correspondingly, it will be interesting to explore the 
potential role of ferroptosis in proton and other high LET radiations.

Together, a comprehensive understanding of these points will allow for further clarification of the mechanisms underlying IR-induced ferroptosis and for more robust establishment of therapeutic strategies targeting ferroptosis in RT, offering opportunities to develop superior FINs for radiosensitization.

\section{ACKNOWLEDGEMENTS}

We apologize to the colleagues whose relevant work cannot be cited in this review due to space limitations. This research has been supported by Radiation Oncology Strategic Initiatives (ROSI) from The University of Texas MD Anderson Cancer Center.

\section{ABBREVIATIONS}

4-HNE, 4-hydroxynonenal; AA, arachidonic acid; ACC, acetyl-CoA carboxylase; ACSL3, acyl coenzyme A synthetase long chain family member 3; ACSL4, acyl coenzyme A synthetase long chain family member 4; AdA, adrenic acid; AIFM2, apoptosis-inducing factorassociated mitochondrial-associated protein 2; ALK, anaplastic lymphoma kinase; ALOX, lipoxygenase; AMPK, AMP-activated protein kinase; ARF, ADP-ribosylation factor; ATF3, activating transcription factor 3; ATF4, activating transcription factor 4; ATM, ataxia telangiectasia mutated; ATR, ataxia telangiectasia and Rad3 related; BAP1, BRCA1 associated protein 1; BAX, BCL2-associated $\mathrm{X}$; $\mathrm{BH} 2$, boron dihydride; $\mathrm{BH} 4$, tetrahydrobiopterin; BRAF, B-Raf proto-oncogene, serine/threonine kinase; $B S O$, buthionine sulphoximine; CARS, cysteinyl-tRNA synthetase; CBS, cystathionine $\beta$ synthase; CDKN1A/p21, cyclin dependent kinase inhibitor $1 \mathrm{~A}$; CHEK1/2, checkpoint kinases1/2; CoA, coenzyme A; CoQ, ubiquinone; CoQH2, ubiquinol; CREB, cAMP-response-element-binding protein; CTLA-4, cytotoxic T-lymphocyte-associated protein 4; CDX, cell line-derived xenograft; DAG, diacylglycerol; DDR, DNA damage response; DFO, desferoxamine; DHFR, dihydrofolate reductase; DPP4, dipeptidyl-peptidase-4; DR 5, death receptor 5; DSB, double strand break; ETC, electron transport chain; FAS, CD95/cluster of differentiation 95; FDXR, ferredoxin reductase; FIN, ferroptosis inducer; FTH1, ferritin heavy chain; FPN1, ferroportin 1; FSP1, ferroptosis suppressor protein 1; $\mathrm{GCH} 1$, guanosine triphosphate cyclohydrolase 1; GFRP, GCH1 feedback regulatory protein; GLS2, glutaminases 2; GPX4, glutathione peroxidase 4; GSH, glutathione; YH2AX, phosphorylated H2A histone family member X; HIF, hypoxia-inducible factor; HILPDA, hypoxia-induced, lipid droplet-associated protein; HO-1, heme oxygenase-1; ICD, immunogenic cell death; ICI, immune checkpoint inhibitor; IFNy, interferon gamma; IR, ionizing radiation; IKE, imidazole ketone erastin; KEAP1, Kelch-like ECH-associated protein 1; LET, linear energy transfer; LPCAT3, lysophosphatidylcholine acyltransferase 3; LysoPL, lysophospholipid; MDA, malondialdehyde; MDM2/MDMX, mouse double minute 2 homolog/murine double minute $\mathrm{X}$; MLKL, mixed lineage kinase domain like pseudokinase; MOMP, mitochondrial outer membrane permeability; MTF1, metal regulatory transcription factor 1; mTOR, mammalian target of rapamycin; MUFA, monounsaturated fatty acid; $\mathrm{NADPH}$, nicotinamide adenine dinucleotide phosphate; NFS1, cysteine desulfurase; NOXA, phorbol-12-myristate-13-acetate- induced protein 1; NRF2, NF-E2-related factor 2; ORR, objective response rate; OTUB1, OTU domain-containing ubiquitin aldehyde binding protein 1 ; p53, tumor protein p53; PAI-1, plasminogen activator inhibitor-1; PDX, patient-derived xenograft; PD-L1, programmed death-ligand 1; PML, promyelocytic leukemia protein; POR, cytochrome P450 oxidoreductase; PTGS2, prostaglandinendoperoxide synthase 2; PUFA-PL, polyunsaturated-fatty-acidcontaining phospholipid; PUMA, p53 upregulated modulator of apoptosis; RB, retinoblastoma; RCD, regulated cell death; RIPK1/ 3 , receptor-interacting serine/threonine protein kinases 1/3; ROS, reactive oxygen species; RT, radiotherapy; SAS, sulfasalazine; SASP, senescence-associated secretory phenotype; SAT1, spermidine/spermine N1-acetyltransferase 1; SCD, stearoyl coenzyme A desaturase; SIRT-1, sirtuin 1; SLC7A11, solute carrier family 7 member 11; SQS, squalene synthase; SSB, single strand break; TFR1, transferrin receptor 1; USP7, ubiquitin-specific protease 7.

\section{AUTHOR CONTRIBUTIONS}

G.L. and B.G. wrote most of the manuscript with assistance from other co-authors. G.L. generated all figures. B.G. supervised and revised the manuscript. All authors commented on the manuscript.

\section{FUNDING}

B.G. is supported by Radiation Oncology Strategic Initiatives (ROSI) from The University of Texas MD Anderson Cancer Center.

\section{CONFLICTS OF INTEREST/COMPETING INTERESTS}

The authors declare that they have no competing interests.

\section{CONSENT TO PARTICIPATE}

This article does not contain any studies with human or animal subjects performed by the any of the authors.

\section{OPEN ACCESS}

This article is licensed under a Creative Commons Attribution 4.0 International License, which permits use, sharing, adaptation, distribution and reproduction in any medium or format, as long as you give appropriate credit to the original author(s) and the source, provide a link to the Creative Commons licence, and indicate if changes were made. The images or other third party material in this article are included in the article's Creative Commons licence, unless indicated otherwise in a credit line to the material. If material is not included in the article's Creative Commons licence and your intended use is not permitted by statutory regulation or exceeds the permitted use, you will need to obtain permission directly from the copyright holder. To view a copy of this licence, visit http:// creativecommons.org/licenses/by/4.0/.

\section{REFERENCES}

Adjemian S, Oltean T, Martens S, Wiernicki B, Goossens V, Berghe TV, Cappe B, Ladik M, Riquet FB, Heyndrickx L (2020) lonizing radiation results in a mixture of cellular outcomes including mitotic 
catastrophe, senescence, methuosis, and iron-dependent cell death. Cell Death Dis 11:1-15

Alim I, Caulfield JT, Chen Y, Swarup V, Geschwind DH, Ivanova E, Seravalli J, Ai Y, Sansing LH, Marie EJS (2019) Selenium drives a transcriptional adaptive program to block ferroptosis and treat stroke. Cell 177(1262-1279):

Alvarez SW, Sviderskiy VO, Terzi EM, Papagiannakopoulos T, Moreira AL, Adams S, Sabatini DM, Birsoy K, Possemato R (2017) NFS1 undergoes positive selection in lung tumours and protects cells from ferroptosis. Nature 551:639-643

Anderson GJ, Vulpe CD (2009) Mammalian iron transport. Cell Mol Life Sci 66:3241

Angeli JPF, Conrad M (2018) Selenium and GPX4, a vital symbiosis. Free Radical Biol Med 127:153-159

Angeli JPF, Schneider M, Proneth B, Tyurina YY, Tyurin VA, Hammond VJ, Herbach N, Aichler M, Walch A, Eggenhofer E (2014) Inactivation of the ferroptosis regulator Gpx4 triggers acute renal failure in mice. Nat Cell Biol 16:1180-1191

Aubrey BJ, Kelly GL, Janic A, Herold MJ, Strasser A (2018) How does p53 induce apoptosis and how does this relate to p53mediated tumour suppression? Cell Death Differ 25:104-113

Ayala A, Muñoz MF, Argüelles S (2014). Lipid peroxidation: production, metabolism, and signaling mechanisms of malondialdehyde and 4-hydroxy-2-nonenal. Oxid Med Cell Longevity

Azzam El, Jay-Gerin J-P, Pain D (2012) lonizing radiation-induced metabolic oxidative stress and prolonged cell injury. Cancer Lett 327:48-60

Badgley MA, Kremer DM, Maurer HC, DelGiorno KE, Lee H-J, Purohit V, Sagalovskiy IR, Ma A, Kapilian J, Firl CE (2020) Cysteine depletion induces pancreatic tumor ferroptosis in mice. Science 368:85-89

Baidoo KE, Yong K, Brechbiel MW (2013) Molecular pathways: targeted a-particle radiation therapy. Clin Cancer Res 19:530537

Benveniste MF, Gomez D, Carter BW, Betancourt Cuellar SL, Shroff GS, Benveniste APA, Odisio EG, Marom EM (2019) Recognizing radiation therapy-related complications in the chest. Radiographics 39:344-366

Berbee M, Fu Q, Boerma M, Pathak R, Zhou D, Kumar KS, HauerJensen M (2011) Reduction of radiation-induced vascular nitrosative stress by the vitamin $E$ analog $y$-tocotrienol: evidence of a role for tetrahydrobiopterin. Int J Radiat Oncol Biol Phys 79:884-891

Bersuker K, Hendricks J, Li Z, Magtanong L, Ford B, Tang PH, Roberts MA, Tong B, Maimone TJ, Zoncu R (2019) The CoQ oxidoreductase FSP1 acts parallel to GPX4 to inhibit ferroptosis. Nature 575:688

Bieging KT, Mello SS, Attardi LD (2014) Unravelling mechanisms of p53-mediated tumour suppression. Nat Rev Cancer 14:359-370

Boumahdi S, de Sauvage FJ (2020) The great escape: tumour cell plasticity in resistance to targeted therapy. Nat Rev Drug Discov 19:39-56

Braig M, Lee S, Loddenkemper C, Rudolph C, Peters AH, Schlegelberger B, Stein H, Dörken B, Jenuwein T, Schmitt CA (2005) Oncogene-induced senescence as an initial barrier in lymphoma development. Nature 436:660-665
Bristol ML, Di X, Beckman MJ, Wilson EN, Henderson SC, Maiti A, Fan Z, Gewirtz DA (2012) Dual functions of autophagy in the response of breast tumor cells to radiation: cytoprotective autophagy with radiation alone and cytotoxic autophagy in radiosensitization by vitamin D3. Autophagy 8:739-753

Brown CW, Amante JJ, Chhoy P, Elaimy AL, Liu H, Zhu LJ, Baer CE, Dixon SJ, Mercurio AM (2019) Prominin2 drives ferroptosis resistance by stimulating iron export. Dev Cell 51(575-586):

Bump EA, Brown JM (1990) Role of glutathione in the radiation response of mammalian cells invitro and in vivo. Pharmacol Ther 47:117-136

Cheema AK, Pathak R, Zandkarimi F, Kaur P, Alkhalil L, Singh R, Zhong X, Ghosh S, Aykin-Burns N, Hauer-Jensen M (2014) Liver metabolomics reveals increased oxidative stress and fibrogenic potential in gfrp transgenic mice in response to ionizing radiation. J Proteome Res 13:3065-3074

Chen C-Y, Oliner JD, Zhan Q, Fornace AJ, Vogelstein B, Kastan MB (1994) Interactions between p53 and MDM2 in a mammalian cell cycle checkpoint pathway. Proc Natl Acad Sci 91:2684-2688

Chen D, Fan Z, Rauh M, Buchfelder M, Eyupoglu I, Savaskan N (2017a) ATF4 promotes angiogenesis and neuronal cell death and confers ferroptosis in a xCT-dependent manner. Oncogene 36:5593-5608

Chen D, Tavana O, Chu B, Erber L, Chen Y, Baer R, Gu W (2017b) NRF2 is a major target of ARF in p53-independent tumor suppression. Mol Cell 68(224-232):

Chen P-H, Wu J, Ding C-KC, Lin C-C, Pan S, Bossa N, Xu Y, Yang $\mathrm{W}-\mathrm{H}$, Mathey-Prevot B, Chi J-T (2020) Kinome screen of ferroptosis reveals a novel role of ATM in regulating iron metabolism. Cell Death Differ 27:1008-1022

Chew SH, Okazaki Y, Akatsuka S, Wang S, Jiang L, Ohara Y, Ito F, Saya H, Sekido Y, Toyokuni S (2017) Rheostatic CD44 isoform expression and its association with oxidative stress in human malignant mesothelioma. Free Radical Biol Med 106:91-99

Chio IIC, Tuveson DA (2017) ROS in cancer: the burning question. Trends Mol Med 23:411-429

Choudhary S, Burns SC, Mirsafian H, Li W, Vo DT, Qiao M, Lei X, Smith AD, Penalva LO (2020) Genomic analyses of early responses to radiation inglioblastoma reveal new alterations at transcription, splicing, and translation levels. Sci Rep 10:1-12

Chu B, Kon N, Chen D, Li T, Liu T, Jiang L, Song S, Tavana O, Gu W (2019) ALOX12 is required for p53-mediated tumour suppression through a distinct ferroptosis pathway. Nat Cell Biol 21:579-591

Colles SM, Chisolm GM (2000) Lysophosphatidylcholine-induced cellular injury in cultured fibroblasts involves oxidative events. J Lipid Res 41:1188-1198

Conrad M, Pratt DA (2019) The chemical basis of ferroptosis. Nat Chem Biol 15:1137-1147

Conrad M, Proneth B (2020) Selenium: tracing another essential element of ferroptotic cell death. Cell Chem Biol

Conrad M, Sato H (2012) The oxidative stress-inducible cystine/ glutamate antiporter, system x c-: cystine supplier and beyond. Amino Acids 42:231-246

Crabtree MJ, Tatham AL, Hale AB, Alp NJ, Channon KM (2009) Critical role for tetrahydrobiopterin recycling by dihydrofolate reductase in regulation of endothelial nitric-oxide synthase 
coupling relative importance of the de novo biopterin synthesis versus salvage pathways. J Biol Chem 284:28128-28136

de la Vega MR, Chapman E, Zhang DD (2018) NRF2 and the hallmarks of cancer. Cancer Cell 34:21-43

Delaney G, Jacob S, Featherstone C, Barton M (2005) The role of radiotherapy in cancer treatment: estimating optimal utilization from a review of evidence-based clinical guidelines. Cancer 104:1129-1137

Dixon SJ, Lemberg KM, Lamprecht MR, Skouta R, Zaitsev EM, Gleason CE, Patel DN, Bauer AJ, Cantley AM, Yang WS (2012) Ferroptosis: an iron-dependent form of nonapoptotic cell death. Cell 149:1060-1072

Dixon SJ, Patel DN, Welsch M, Skouta R, Lee ED, Hayano M, Thomas AG, Gleason CE, Tatonetti NP, Slusher BS (2014) Pharmacological inhibition of cystine-glutamate exchange induces endoplasmic reticulum stress and ferroptosis. Elife 3:

Dixon SJ, Winter GE, Musavi LS, Lee ED, Snijder B, Rebsamen M, Superti-Furga G, Stockwell BR (2015) Human haploid cell genetics reveals roles for lipid metabolism genes in nonapoptotic cell death. ACS Chem Biol 10:1604-1609

Doll S, Proneth B, Tyurina YY, Panzilius E, Kobayashi S, Ingold I, Irmler M, Beckers J, Aichler M, Walch A (2017) ACSL4 dictates ferroptosis sensitivity by shaping cellular lipid composition. Nat Chem Biol 13:91-98

Doll S, Freitas FP, Shah R, Aldrovandi M, da Silva MC, Ingold I, Grocin AG, da Silva TNX, Panzilius E, Scheel C (2019) FSP1 is a glutathione-independent ferroptosis suppressor. Nature. 575:693

Duberley K, Heales S, Abramov A, Chalasani A, Land J, Rahman S, Hargreaves I (2014) Effect of Coenzyme Q10 supplementation on mitochondrial electron transport chain activity and mitochondrial oxidative stress in Coenzyme Q10 deficient human neuronal cells. Int J Biochem Cell Biol 50:60-63

Elguindy MM, Nakamaru-Ogiso E (2015) Apoptosis-inducing factor (AIF) and its family member protein, AMID, are rotenonesensitive NADH: ubiquinone oxidoreductases (NDH-2). J Biol Chem 290:20815-20826

Fan Z, Wirth A, Chen D, Wruck C, Rauh M, Buchfelder M, Savaskan N (2017) Nrf2-Keap1 pathway promotes cell proliferation and diminishes ferroptosis. Oncogenesis 6:e371-e371

Fei P, El-Deiry WS (2003) P53 and radiation responses. Oncogene 22:5774-5783

Feng H, Schorpp K, Jin J, Yozwiak CE, Hoffstrom BG, Decker AM, Rajbhandari P, Stokes ME, Bender HG, Csuka JM (2020) Transferrin receptor is a specific ferroptosis marker. Cell Rep 30(3411-3423):

Frei B, Kim MC, Ames BN (1990) Ubiquinol-10 is an effective lipidsoluble antioxidant at physiological concentrations. Proc Natl Acad Sci 87:4879-4883

Galluzzi L, Vitale I, Aaronson SA, Abrams JM, Adam D, Agostinis P, Alnemri ES, Altucci L, Amelio I, Andrews DW (2018) Molecular mechanisms of cell death: recommendations of the Nomenclature Committee on Cell Death 2018. Cell Death Differ 25:486541

Gao M, Monian P, Quadri N, Ramasamy R, Jiang X (2015) Glutaminolysis and transferrin regulate ferroptosis. Mol Cell 59:298-308
Gao M, Monian P, Pan Q, Zhang W, Xiang J, Jiang X (2016) Ferroptosis is an autophagic cell death process. Cell Res 26:1021-1032

Garcia-Bermudez J, Baudrier L, Bayraktar EC, Shen Y, La K, Guarecuco R, Yucel B, Fiore D, Tavora B, Freinkman E (2019) Squalene accumulation in cholesterol auxotrophic lymphomas prevents oxidative cell death. Nature 567:118-122

Geng N, Shi B, Li S, Zhong Z, Li Y, Xua W, Zhou H, Cai J (2018) Knockdown of ferroportin accelerates erastin-induced ferroptosis in neuroblastoma cells. Eur Rev Med Pharmacol Sci 22:38263836

Georgakilas AG, Martin OA, Bonner WM (2017) p21: a two-faced genome guardian. Trends Mol Med 23:310-319

Green DR (2019) The coming decade of cell death research: five riddles. Cell 177:1094-1107

Gudkov AV, Komarova EA (2003) The role of p53 in determining sensitivity to radiotherapy. Nat Rev Cancer 3:117-129

Guo J, Xu B, Han Q, Zhou H, Xia Y, Gong C, Dai X, Li Z, Wu G (2018) Ferroptosis: a novel anti-tumor action for cisplatin. Cancer Res Treat 50:445

Habib E, Linher-Melville K, Lin H-X, Singh G (2015) Expression of xCT and activity of system xc- are regulated by NRF2 in human breast cancer cells in response to oxidative stress. Redox Biol 5:33-42

Han Y, Platonov A, Akhalaia M, Yun Y-S, Song J-Y (2005) Differential effect of $Y$-radiation-induced heme oxygenase-1 activity in female and male C57BL/6 mice. J Korean Med Sci 20:535-541

Hanahan D, Weinberg RA (2011) Hallmarks of cancer: the next generation. Cell 144:646-674

Hangauer MJ, Viswanathan VS, Ryan MJ, Bole D, Eaton JK, Matov A, Galeas J, Dhruv HD, Berens ME, Schreiber SL (2017) Drugtolerant persister cancer cells are vulnerable to GPX4 inhibition. Nature 551:247-250

Hassannia B, Wiernicki B, Ingold I, Qu F, Van Herck S, Tyurina YY, Bayır H, Abhari BA, Angeli JPF, Choi SM (2018) Nano-targeted induction of dual ferroptotic mechanisms eradicates high-risk neuroblastoma. J Clin Investig 128:3341-3355

Hassannia B, Vandenabeele P, Berghe TV (2019) Targeting ferroptosis to iron out cancer. Cancer Cell 35:830-849

Hayano M, Yang W, Corn C, Pagano N, Stockwell B (2016) Loss of cysteinyl-tRNA synthetase (CARS) induces the transsulfuration pathway and inhibits ferroptosis induced by cystine deprivation. Cell Death Differ 23:270-278

Herrera FG, Bourhis J, Coukos G (2017) Radiotherapy combination opportunities leveraging immunity for the next oncology practice. Cancer J Clin 67:65-85

Horikoshi N, Cong J, Kley N, Shenk T (1999) Isolation of differentially expressed cDNAs from p53-dependent apoptotic cells: activation of the human homologue of the Drosophila peroxidasin gene. Biochem Biophys Res Commun 261:864-869

Hou W, Xie Y, Song X, Sun X, Lotze MT, Zeh HJ III, Kang R, Tang D (2016) Autophagy promotes ferroptosis by degradation of ferritin. Autophagy 12:1425-1428

Hu W, Zhang C, Wu R, Sun Y, Levine A, Feng Z (2010) Glutaminase 2 , a novel p53 target gene regulating energy metabolism and antioxidant function. Proc Natl Acad Sci 107:7455-7460 
Hu L, Wang H, Huang L, Zhao Y, Wang J (2016) Crosstalk between autophagy and intracellular radiation response. Int $\mathrm{J}$ Oncol 49:2217-2226

Hu K, Li K, Lv J, Feng J, Chen J, Wu H, Cheng F, Jiang W, Wang J, Pei H (2020) Suppression of the SLC7A11/glutathione axis causes synthetic lethality in KRAS-mutant lung adenocarcinoma. $\mathrm{J}$ Clin Invest 130:1752

Huang R-X, Zhou P-K (2020) DNA damage response signaling pathways and targets for radiotherapy sensitization in cancer. Signal Transd Target Therapy 5:1-27

Hwang PM, Bunz F, Yu J, Rago C, Chan TA, Murphy MP, Kelso GF, Smith RA, Kinzler KW, Vogelstein B (2001) Ferredoxin reductase affects p53-dependent, 5-fluorouracil-induced apoptosis in colorectal cancer cells. Nat Med 7:1111-1117

Ingold I, Berndt C, Schmitt S, Doll S, Poschmann G, Buday K, Roveri A, Peng X, Freitas FP, Seibt T (2018) Selenium utilization by GPX4 is required to prevent hydroperoxide-induced ferroptosis. Cell 172(409-422):

Ishimoto T, Nagano O, Yae T, Tamada M, Motohara T, Oshima H, Oshima M, Ikeda T, Asaba R, Yagi H (2011) CD44 variant regulates redox status in cancer cells by stabilizing the $\mathrm{XCT}$ subunit of system $\mathrm{xc}^{-}$and thereby promotes tumor growth. Cancer Cell 19:387-400

Jaffray DA (2012) Image-guided radiotherapy: from current concept to future perspectives. Nat Rev Clin Oncol 9:688

Jiang L, Kon N, Li T, Wang S-J, Su T, Hibshoosh H, Baer R, Gu W (2015) Ferroptosis as a p53-mediated activity during tumour suppression. Nature 520:57-62

Kagan VE, Mao G, Qu F, Angeli JPF, Doll S, St Croix C, Dar HH, Liu B, Tyurin VA, Ritov VB (2017) Oxidized arachidonic and adrenic PEs navigate cells to ferroptosis. Nat Chem Biol 13:81-90

Kaku Y, Tsuchiya A, Kanno T, Nishizaki T (2015) HUHS1015 induces necroptosis and caspase-independent apoptosis of MKN28 human gastric cancer cells in association with AMID accumulation in the nucleus. Anti-Cancer Agents Med Chem 15:242-247

Kam WW-Y, Banati RB (2013) Effects of ionizing radiation on mitochondria. Free Radical Biol Med 65:607-619

Kang R, Tang D (2016) What is the pathobiology of inflammation to cell death? Apoptosis, necrosis, necroptosis, autophagic cell death, pyroptosis, and NETosis. In: Autophagy networks in inflammation. Springer, Berlin, pp 81-106

Kang R, Kroemer G, Tang D (2019) The tumor suppressor protein p53 and the ferroptosis network. Free Radical Biol Med 133:162168

Kim SE, Zhang L, Ma K, Riegman M, Chen F, Ingold I, Conrad M, Turker MZ, Gao M, Jiang X (2016) Ultrasmall nanoparticles induce ferroptosis in nutrient-deprived cancer cells and suppress tumour growth. Nat Nanotechnol 11:977

Koppula P, Zhang Y, Zhuang L, Gan B (2018) Amino acid transporter SLC7A11/xCT at the crossroads of regulating redox homeostasis and nutrient dependency of cancer. Cancer Commun 38:1-13

Koppula P, Zhuang L, Gan B (2020) Cystine transporter SLC7A11/ XCT in cancer: ferroptosis, nutrient dependency, and cancer therapy. Protein Cell. https://doi.org/10.1007/s13238-020-007895

Kordbacheh T, Honeychurch J, Blackhall F, Faivre-Finn C, Illidge T (2018) Radiotherapy and anti-PD-1/PD-L1 combinations in lung cancer: building better translational research platforms. Ann Oncol 29:301-310

Kraft VA, Bezjian CT, Pfeiffer S, Ringelstetter L, Müller C, Zandkarimi F, Merl-Pham J, Bao X, Anastasov N, Kössl J (2019) GTP cyclohydrolase $1 /$ tetrahydrobiopterin counteract ferroptosis through lipid remodeling. ACS Central Sci 6:41-53

Lang X, Green MD, Wang W, Yu J, Choi JE, Jiang L, Liao P, Zhou J, Zhang Q, Dow A (2019) Radiotherapy and immunotherapy promote tumoral lipid oxidation and ferroptosis via synergistic repression of SLC7A11. Cancer Discov 9:1673-1685

Leach JK, Van Tuyle G, Lin P-S, Schmidt-Ullrich R, Mikkelsen RB (2001) lonizing radiation-induced, mitochondria-dependent generation of reactive oxygen/nitrogen. Cancer Res 61:3894-3901

Lee H, Zandkarimi F, Zhang Y, Meena JK, Kim J, Zhuang L, Tyagi S, Ma L, Westbrook TF, Steinberg GR (2020) Energy-stressmediated AMPK activation inhibits ferroptosis. Nat Cell Biol 22:225-234

Lei G, Zhang Y, Koppula P, Liu X, Zhang J, Lin SH, Ajani JA, Xiao Q, Liao Z, Wang H (2020) The role of ferroptosis in ionizing radiation-induced cell death and tumor suppression. Cell Res 30:146-162

Li L, Rezvan A, Salerno JC, Husain A, Kwon K, Jo H, Harrison DG, Chen W (2010) GTP cyclohydrolase I phosphorylation and interaction with GTP cyclohydrolase feedback regulatory protein provide novel regulation of endothelial tetrahydrobiopterin and nitric oxide. Circ Res 106:328-336

Li M, You L, Xue J, Lu Y (2018) lonizing radiation-induced cellular senescence in normal, non-transformed cells and the involved DNA damage response: a mini review. Front Pharmacol 9:522

Li X, Duan L, Yuan S, Zhuang X, Qiao T, He J (2019a) Ferroptosis inhibitor alleviates radiation-induced lung fibrosis (RILF) via down-regulation of TGF- $\beta 1$. J Inflamm 16:11

Li X, Zhuang X, Qiao T (2019b) Role of ferroptosis in the process of acute radiation-induced lung injury in mice. Biochem Biophys Res Commun 519:240-245

Li C, Dong X, Du W, Shi X, Chen K, Zhang W, Gao M (2020) LKB1AMPK axis negatively regulates ferroptosis by inhibiting fatty acid synthesis. Signal Transd Target Ther 5:1-4

Liang C, Zhang X, Yang M, Dong X (2019) Recent progress in ferroptosis inducers for cancer therapy. Adv Mater 31:1904197

Liu B, Yi J, Yang X, Liu L, Lou X, Zhang Z, Qi H, Wang Z, Zou J, Zhu W-G (2019a) MDM2-mediated degradation of WRN promotes cellular senescence in a p53-independent manner. Oncogene 38:2501-2515

Liu T, Jiang L, Tavana O, Gu W (2019b) The deubiquitylase OTUB1 mediates ferroptosis via stabilization of SLC7A11. Cancer Res 79:1913-1924

Liu J, Kuang F, Kroemer G, Klionsky DJ, Kang R, Tang D (2020a) Autophagy-dependent ferroptosis: machinery and regulation. Cell Chem Biol 27:420

Liu J, Zhu Z, Liu Y, Wei L, Li B, Mao F, Zhang J, Wang Y, Liu Y (2020b) MDM2 inhibition-mediated autophagy contributes to the pro-apoptotic effect of berberine in p53-null leukemic cells. Life Sci 242:

Liu X, Olszewski K, Zhang Y, Lim EW, Shi J, Zhang X, Zhang J, Lee $H$, Koppula P, Lei $G$ (2020c) Cystine transporter regulation of pentose phosphate pathway dependency and disulfide stress 
exposes a targetable metabolic vulnerability in cancer. Nat Cell Biol 22:476-486

Liu X, Zhang Y, Zhuang L, Olszewski K, Gan B (2020d) NADPH debt drives redox bankruptcy: SLC7A11/xCT-mediated cystine uptake as a double-edge sword in cellular redox regulation. Genes Dis. https://doi.org/10.1016/j.gendis.2020.11.010

Louandre C, Marcq I, Bouhlal H, Lachaier E, Godin C, Saidak Z, François C, Chatelain D, Debuysscher V, Barbare J-C (2015) The retinoblastoma $(\mathrm{Rb})$ protein regulates ferroptosis induced by sorafenib in human hepatocellular carcinoma cells. Cancer Lett 356:971-977

Ma S, Henson E, Chen Y, Gibson S (2016) Ferroptosis is induced following siramesine and lapatinib treatment of breast cancer cells. Cell Death Dis 7:e2307-e2307

Magtanong L, Ko P-J, To M, Cao JY, Forcina GC, Tarangelo A, Ward CC, Cho K, Patti GJ, Nomura DK (2019) Exogenous monounsaturated fatty acids promote a ferroptosis-resistant cell state. Cell Chem Biol 26(420-432):

Maier P, Hartmann L, Wenz F, Herskind C (2016) Cellular pathways in response to ionizing radiation and their targetability for tumor radiosensitization. Int J Mol Sci 17:102

Malhotra J, Jabbour SK, Aisner J (2017) Current state of immunotherapy for non-small cell lung cancer. Transl Lung Cancer Res 6:196

Marshall KR, Gong M, Wodke L, Lamb JH, Jones DJ, Farmer PB, Scrutton NS, Munro AW (2005) The human apoptosis-inducing protein AMID is an oxidoreductase with a modified flavin cofactor and DNA binding activity. J Biol Chem 280:30735-30740

Maya R, Balass M, Kim S-T, Shkedy D, Leal J-FM, Shifman O, Moas M, Buschmann T, Ronai ZE, Shiloh Y (2001) ATM-dependent phosphorylation of Mdm2 on serine 395: role in p53 activation by DNA damage. Genes Dev 15:1067-1077

McCullagh EA, Featherstone DE (2014) Behavioral characterization of system xc-mutant mice. Behav Brain Res 265:1-11

McDonald JT, Kim K, Norris AJ, Vlashi E, Phillips TM, Lagadec C, Della Donna L, Ratikan J, Szelag H, Hlatky L (2010) lonizing radiation activates the Nrf2 antioxidant response. Cancer Res 70:8886-8895

Mijit M, Caracciolo V, Melillo A, Amicarelli F, Giordano A (2020) Role of $p 53$ in the regulation of cellular senescence. Biomolecules 10:420

Mohamad O, Tabuchi T, Nitta Y, Nomoto A, Sato A, Kasuya G, Makishima H, Choy H, Yamada S, Morishima T (2019) Risk of subsequent primary cancers after carbon ion radiotherapy, photon radiotherapy, or surgery for localised prostate cancer: a propensity score-weighted, retrospective, cohort study. Lancet Oncol 20:674-685

Mohan R, Grosshans D (2017) Proton therapy: present and future. Adv Drug Deliv Rev 109:26-44

Mumbauer S, Pascual J, Kolotuev I, Hamaratoglu F (2019) Ferritin heavy chain protects the developing wing from reactive oxygen species and ferroptosis. PLoS Genet 15:

Nehs MA, Lin C-I, Kozono DE, Whang EE, Cho NL, Zhu K, Moalem J, Moore FD Jr, Ruan DT (2011) Necroptosis is a novel mechanism of radiation-induced cell death in anaplastic thyroid and adrenocortical cancers. Surgery 150:1032-1039
Nguyen HP, Yi D, Lin F, Viscarra JA, Tabuchi C, Ngo K, Shin G et al (2020) Aifm2, a NADH oxidase, supports robust glycolysis and is required for cold-and diet-induced thermogenesis. Mol Cell 77 (600-617):

Ohiro Y, Garkavtsev I, Kobayashi S, Sreekumar KR, Nantz R, Higashikubo BT, Duffy SL, Higashikubo R, Usheva A, Gius D (2002) A novel p53-inducible apoptogenic gene, PRG3, encodes a homologue of the apoptosis-inducing factor (AIF). FEBS Lett 524:163-171

Ou Y, Wang S-J, Li D, Chu B, Gu W (2016) Activation of SAT1 engages polyamine metabolism with p53-mediated ferroptotic responses. Proc Natl Acad Sci 113:E6806-E6812

Padanad MS, Konstantinidou G, Venkateswaran N, Melegari M, Rindhe S, Mitsche M, Yang C, Batten K, Huffman KE, Liu J (2016) Fatty acid oxidation mediated by Acyl-CoA synthetase long chain 3 is required for mutant KRAS lung tumorigenesis. Cell Rep 16:1614-1628

Pan X, Lin Z, Jiang D, Yu Y, Yang D, Zhou H, Zhan D, Liu S, Peng G, Chen $Z$ (2019) Erastin decreases radioresistance of NSCLC cells partially by inducing GPX4-mediated ferroptosis. Oncol Lett 17:3001-3008

Pang M, Liu X, Slagle-Webb B, Madhankumar A, Connor J (2016) Role of $h$-ferritin in radiosensitivity of human gliomacells. J Cancer Biol Treat 3:1-10

Pathak R, Pawar SA, Fu Q, Gupta PK, Berbée M, Garg S, Sridharan V, Wang W, Biju PG, Krager KJ (2014) Characterization of transgenic Gfrp knock-in mice: implications for tetrahydrobiopterin in modulation of normal tissue radiation responses. Antioxid Redox Signal 20:1436-1446

Paton CM, Ntambi JM (2009) Biochemical and physiological function of stearoyl-CoA desaturase. Am J Physiol Endocrinol Metab 297: E28-E37

Ran $\mathrm{Q}$, Liang $\mathrm{H}$, Ikeno $\mathrm{Y}, \mathrm{Qi}$ W, Prolla TA, Roberts LJ, Wolf N, VanRemmen H, Richardson A (2007) Reduction in glutathione peroxidase 4 increases life span through increased sensitivity to apoptosis. J Gerontol A 62:932-942

Rao SG, Jackson JG (2016) SASP: tumor suppressor or promoter? Yes! Trends Cancer 2:676-687

Reisz JA, Bansal N, Qian J, Zhao W, Furdui CM (2014) Effects of ionizing radiation on biological molecules-mechanisms of damage and emerging methods of detection. Antioxid Redox Signal 21:260-292

Sabin RJ, Anderson RM (2011) Cellular Senescence-its role in cancer and the response to ionizing radiation. Genome Integrity 2:7

Sanli T, Rashid A, Liu C, Harding S, Bristow RG, Cutz J-C, Singh G, Wright J, Tsakiridis T (2010) lonizing radiation activates AMPactivated kinase (AMPK): a target for radiosensitization of human cancer cells. Int J Radiat Oncol Biol Phys 78:221-229

Sanli T, Steinberg GR, Singh G, Tsakiridis T (2014) AMP-activated protein kinase (AMPK) beyond metabolism: a novel genomic stress sensor participating in the DNA damage response pathway. Cancer Biol Ther 15:156-169

Sato H, Tamba M, Ishii T, Bannai S (1999) Cloning and expression of a plasma membrane cystine/glutamate exchange transporter composed of two distinct proteins. J Biol Chem 274:11455-11458 
Sato H, Shiiya A, Kimata M, Maebara K, Tamba M, Sakakura Y, Makino N, Sugiyama F, Yagami K-I, Moriguchi T (2005) Redox imbalance in cystine/glutamate transporter-deficient mice. J Biol Chem 280:37423-37429

Shadyro O, Yurkova I, Kisel M (2002) Radiation-induced peroxidation and fragmentation of lipids in a model membrane. Int $\mathrm{J}$ Radiat Biol 78:211-217

Shah R, Shchepinov MS, Pratt DA (2018) Resolving the role of lipoxygenases in the initiation and execution of ferroptosis. ACS Central Sci 4:387-396

Sheikh M, Fornace AJ (2000) Death and decoy receptors and p53mediated apoptosis. Leukemia 14:1509-1513

Shimada K, Skouta R, Kaplan A, Yang WS, Hayano M, Dixon SJ, Brown LM, Valenzuela CA, Wolpaw AJ, Stockwell BR (2016) Global survey of cell death mechanisms reveals metabolic regulation of ferroptosis. Nat Chem Biol 12:497-503

Singhal R, Mitta SR, Olive KP, Lyssiotis CA, Shah YM (2019) Hypoxia inducible factor- $2 \alpha$ increases sensitivity of colon cancer cells towards oxidative cell death. BioRxiv, 823997

Song YP, Colaco RJ (2018) Radiation necrosis-a growing problem in a case of brain metastases following whole brain radiotherapy and stereotactic radiosurgery. Cureus 10

Song X, Zhu S, Chen P, Hou W, Wen Q, Liu J, Xie Y, Liu J, Klionsky DJ, Kroemer G (2018) AMPK-mediated BECN1 phosphorylation promotes ferroptosis by directly blocking system Xc-activity. Curr Biol 28(2388-2399):

Soula M, Weber RA, Zilka O, Alwaseem H, La K, Yen F, Molina H, Garcia-Bermudez J, Pratt DA, Birsoy K (2020) Metabolic determinants of cancer cell sensitivity to canonical ferroptosis inducers. Nat Chem Biol 16:1351-1360

Stockwell BR, Angeli JPF, Bayir H, Bush Al, Conrad M, Dixon SJ, Fulda S, Gascón S, Hatzios SK, Kagan VE (2017) Ferroptosis: a regulated cell death nexus linking metabolism, redox biology, and disease. Cell 171:273-285

Stockwell BR, Jiang X, Gu W (2020) Emerging mechanisms and disease relevance of ferroptosis. Trends Cell Biol

Sun X, Niu X, Chen R, He W, Chen D, Kang R, Tang D (2016) Metallothionein-1G facilitates sorafenib resistance through inhibition of ferroptosis. Hepatology 64:488-500

Suzuki S, Tanaka T, Poyurovsky MV, Nagano H, Mayama T, Ohkubo S, Lokshin M, Hosokawa H, Nakayama T, Suzuki Y (2010) Phosphate-activated glutaminase (GLS2), a p53-inducible regulator of glutamine metabolism and reactive oxygen species. Proc Natl Acad Sci 107:7461-7466

Tarangelo A, Magtanong L, Bieging-Rolett KT, Li Y, Ye J, Attardi LD, Dixon SJ (2018) p53 suppresses metabolic stress-induced ferroptosis in cancer cells. Cell Rep 22:569-575

Tesfay L, Paul BT, Konstorum A, Deng Z, Cox AO, Lee J, Furdui CM, Hegde P, Torti FM, Torti SV (2019) Stearoyl-coa desaturase 1 protects ovarian cancer cells from ferroptotic cell death. Cancer Res 79:5355-5366

Thariat J, Hannoun-Levi J-M, Myint AS, Vuong T, Gérard J-P (2013) Past, present, and future of radiotherapy for the benefit of patients. Nat Rev Clin Oncol 10:52

Tsoi J, Robert L, Paraiso K, Galvan C, Sheu KM, Lay J, Wong DJ, Atefi M, Shirazi R, Wang X (2018) Multi-stage differentiation defines melanoma subtypes with differential vulnerability to drug- induced iron-dependent oxidative stress. Cancer Cell 33(890904):

Vakifahmetoglu H, Olsson M, Zhivotovsky B (2008) Death through a tragedy: mitotic catastrophe. Cell Death Differ 15:1153-1162

Vařecha $M$, Amrichová J, Zimmermann $M$, Ulman $V$, Lukášová $E$, Kozubek M (2007) Bioinformatic and image analyses of the cellular localization of the apoptotic proteins endonuclease G, AIF, and AMID during apoptosis in human cells. Apoptosis 12:1155-1171

Venkatesh D, O'Brien NA, Zandkarimi F, Tong DR, Stokes ME, Dunn DE, Kengmana ES, Aron AT, Klein AM, Csuka JM (2020) MDM2 and MDMX promote ferroptosis by PPARa-mediated lipid remodeling. Genes Dev 34:526-543

Viswanathan VS, Ryan MJ, Dhruv HD, Gill S, Eichhoff OM, Seashore-Ludlow B, Kaffenberger SD, Eaton JK, Shimada K, Aguirre AJ (2017) Dependency of a therapy-resistant state of cancer cells on a lipid peroxidase pathway. Nature 547:453-457

Vousden KH (2000) p53: death star. Cell 103:691-694

Walden T, Hughes H (1988) SpringerLink (Online service). Prostaglandin and lipid metabolism in radiation injury. Springer, Boston

Wang L, Cai H, Hu Y, Liu F, Huang S, Zhou Y, Yu J, Xu J, Wu F (2018) A pharmacological probe identifies cystathionine $\beta$ synthase as a new negative regulator for ferroptosis. Cell Death Dis 9:1-17

Wang $\mathrm{H}$, Jiang $\mathrm{H}$, Van De Gucht M, De Ridder M (2019a) Hypoxic radioresistance: can ROS be the key to overcome it? Cancers 11:112

Wang W, Green M, Choi JE, Gijón M, Kennedy PD, Johnson JK, Liao P, Lang X, Kryczek I, Sell A (2019b) CD8+ T cells regulate tumour ferroptosis during cancer immunotherapy. Nature 569:270-274

Wang Y, Yang L, Zhang X, Cui W, Liu Y, Sun QR, He Q, Zhao S, Zhang GA, Wang $Y$ (2019c) Epigenetic regulation of ferroptosis by $\mathrm{H} 2 \mathrm{~B}$ monoubiquitination and p53. EMBO Rep 20:

Wang L, Liu Y, Du T, Yang H, Lei L, Guo M, Ding H-F, Zhang J, Wang $H$, Chen X (2020) ATF3 promotes erastin-induced ferroptosis by suppressing system Xc-. Cell Death Differ 27:662-675

Wenzel SE, Tyurina YY, Zhao J, Croix CMS, Dar HH, Mao G, Tyurin VA, Anthonymuthu TS, Kapralov AA, Amoscato AA (2017) PEBP1 wardens ferroptosis by enabling lipoxygenase generation of lipid death signals. Cell 171(628-641):

Williams MV, James ND, Summers E, Barrett A, Ash DV, SubCommittee A (2006) National survey of radiotherapy fractionation practice in 2003. Clin Oncol 18:3-14

Withers HR (1985) Biologic basis for altered fractionation schemes. Cancer 55:2086-2095

Wolszczak M, Gajda J (2010) Iron release from ferritin induced by light and ionizing radiation. Res Chem Intermed 36:549-563

Woo JH, Shimoni Y, Yang WS, Subramaniam P, lyer A, Nicoletti P, Martínez MR, López G, Mattioli M, Realubit R (2015) Elucidating compound mechanism of action by network perturbation analysis. Cell 162:441-451

Wu D, Prives C (2018) Relevance of the p53-MDM2 axis to aging. Cell Death Differ 25:169-179

Wu M, Xu L-G, Li X, Zhai Z, Shu H-B (2002) AMID, an apoptosisinducing factor-homologous mitochondrion-associated protein, 
induces caspase-independent apoptosis. J Biol Chem 277:25617-25623

Wu M, Xu L-G, Su T, Tian Y, Zhai Z, Shu H-B (2004) AMID is a p53inducible gene downregulated in tumors. Oncogene 23:68156819

Xie L, Song X, Yu J, Guo W, Wei L, Liu Y, Wang X (2011) Solute carrier protein family may involve in radiation-induced radioresistance of non-small cell lung cancer. J Cancer Res Clin Oncol 137:1739

Xie Y, Zhu S, Song X, Sun X, Fan Y, Liu J, Zhong M, Yuan H, Zhang L, Billiar TR (2017) The tumor suppressor p53 limits ferroptosis by blocking DPP4 activity. Cell Rep 20:1692-1704

Yan B, Ai Y, Sun Q, Ma Y, Cao Y, Wang J, Zhang Z, Wang X (2020) Membrane damage during ferroptosis is caused by oxidation of phospholipids catalyzed by the oxidoreductases POR and CYB5R1. Mol Cell

Yang D, Yaguchi T, Nagata T, Gotoh A, Dovat S, Song C, Nishizaki T (2011) AMID mediates adenosine-induced caspase-independent HuH-7 cell apoptosis. Cell Physiol Biochem 27:37-44

Yang WS, SriRamaratnam R, Welsch ME, Shimada K, Skouta R, Viswanathan VS, Cheah JH, Clemons PA, Shamji AF, Clish CB (2014) Regulation of ferroptotic cancer cell death by GPX4. Cell 156:317-331

Yang WS, Kim KJ, Gaschler MM, Patel M, Shchepinov MS, Stockwell BR (2016) Peroxidation of polyunsaturated fatty acids by lipoxygenases drives ferroptosis. Proc Natl Acad Sci 113: E4966-E4975

Ye LF, Chaudhary KR, Zandkarimi F, Harken AD, Kinslow CJ, Upadhyayula PS, Dovas A, Higgins DM, Tan H, Zhang Y (2020) Radiation-induced lipid peroxidation triggers ferroptosis and synergizes with ferroptosis inducers. ACS Chem Biol 15:469-484

Yi J, Zhu J, Wu J, Thompson CB, Jiang X (2020) Oncogenic activation of PI3K-AKT-mTOR signaling suppresses ferroptosis via SREBP-mediated lipogenesis. Proc Natl Acad Sci 117:31189-31197

Yoo S-E, Chen L, Na R, Liu Y, Rios C, Van Remmen H, Richardson A, Ran Q (2012) Gpx4 ablation in adult mice results in a lethal phenotype accompanied by neuronal loss in brain. Free Radical Biol Med 52:1820-1827

Zhang D, Wang W, Sun X, Xu D, Wang C, Zhang Q, Wang H, Luo W, Chen $Y$, Chen $\mathrm{H}$ (2016) AMPK regulates autophagy by phosphorylating BECN1 at threonine 388. Autophagy 12:1447-1459
Zhang Y, Qian Y, Zhang J, Yan W, Jung Y-S, Chen M, Huang E, Lloyd K, Duan Y, Wang J (2017) Ferredoxin reductase is critical for p53-dependent tumor suppression via iron regulatory protein 2. Genes Dev 31:1243-1256

Zhang Y, Shi J, Liu X, Feng L, Gong Z, Koppula P, Sirohi K, Li X, Wei Y, Lee H (2018) BAP1 links metabolic regulation of ferroptosis to tumour suppression. Nat Cell Biol 20:1181-1192

Zhang Y, Tan H, Daniels JD, Zandkarimi F, Liu H, Brown LM, Uchida K, O'Connor OA, Stockwell BR (2019a) Imidazole ketone erastin induces ferroptosis and slows tumor growth in a mouse lymphoma model. Cell Chem Biol 26(623-633):

Zhang Y, Zhuang L, Gan B (2019b) BAP1 suppresses tumor development by inducing ferroptosis upon SLC7A11 repression. Mol Cell Oncol 6:1536845

Zhang X, Sui S, Wang L, Li H, Zhang L, Xu S, Zheng X (2020) Inhibition of tumor propellant glutathione peroxidase 4 induces ferroptosis in cancer cells and enhances anticancer effect of cisplatin. J Cell Physiol 235:3425-3437

Zheng J, Conrad M (2020) The metabolic underpinnings of ferroptosis. Cell Metabol

Zhu J, Berisa M, Schwörer S, Qin W, Cross JR, Thompson CB (2019) Transsulfuration activity can support cell growth upon extracellular cysteine limitation. Cell Metab 30(865-876):

Zong Y, Feng S, Yu C, Cheng J, Lu G (2017) Up-regulated ATF4 expression increases cell sensitivity to apoptosis in response to radiation. Cell Physiol Biochem 41:784-794

Zou Y, Schreiber SL (2020) Progress in understanding ferroptosis and challenges in its targeting for therapeutic benefit. Cell Chem Biol 27:463-471

Zou Y, Palte MJ, Deik AA, Li H, Eaton JK, Wang W, Tseng Y-Y, Deasy R, Kost-Alimova M, Dančík V (2019) A GPX4-dependent cancer cell state underlies the clear-cell morphology and confers sensitivity to ferroptosis. Nat Commun 10:1-13

Zou Y, Henry WS, Ricq EL, Graham ET, Phadnis VV, Maretich P, Paradkar S, Boehnke N, Deik AA, Reinhardt F (2020a) Plasticity of ether lipids promotes ferroptosis susceptibility and evasion. Nature 585:603-608

Zou Y, Li H, Graham ET, Deik AA, Eaton JK, Wang W, SandovalGomez G, Clish CB, Doench JG, Schreiber SL (2020b) Cytochrome P450 oxidoreductase contributes to phospholipid peroxidation in ferroptosis. Nat Chem Biol 16:302-309 